

\title{
Minimisation and reduction of 5-coverings of elliptic curves
}

\author{
Tom Fisher
}

\begin{abstract}
We consider models for genus-1 curves of degree 5, which arise in explicit 5descent on elliptic curves. We prove a theorem on the existence of minimal models with the same invariants as the minimal model of the Jacobian elliptic curve and give an algorithm for computing such models. Finally we describe how to reduce genus-1 models of degree 5 defined over $\mathbb{Q}$.
\end{abstract}

\section{Introduction}

Let $E$ be an elliptic curve defined over a number field $K$. An $n$-covering of $E$ is a pair $(C, \pi)$, where $C$ is a smooth curve of genus 1 and $\pi: C \rightarrow E$ is a morphism, both defined over $K$, with the property that $\pi=[n] \circ \psi$ for some isomorphism $\psi: C \rightarrow E$ defined over $\bar{K}$. An $n$-descent on $E$ computes the everywhere locally soluble $n$-coverings of $E$. For such $n$-coverings, we have $\psi^{*}\left(n .0_{E}\right) \sim D$ for some $K$-rational divisor $D$ on $C$. The complete linear system $|D|$ defines a morphism $C \rightarrow \mathbb{P}^{n-1}$. Thus, in the cases $n=2,3,4$, we may represent $C$ by a binary quartic, ternary cubic, or pair of quadrics in four variables. In the case $n=5$, we obtain curves $C \subset \mathbb{P}^{4}$ of degree 5 that are defined by the $4 \times 4$ Pfaffians of a $5 \times 5$ alternating matrix of linear forms.

The question naturally arises as to how we can choose coordinates on $\mathbb{P}^{n-1}$ so that the equations for $C$ have small coefficients. In the cases $n=2,3,4$, this was answered in [Cremona et al. 2010] using the combination of two techniques called minimisation and reduction. In this paper, we extend to the case $n=5$. We establish results on minimisation over an arbitrary local field (immediately implying results over any number field of class number 1), whereas those for reduction are specific to the case $K=\mathbb{Q}$. Implementations of our algorithms in the case $K=\mathbb{Q}$ are available in Magma [Bosma et al. 1997].

MSC2010: primary $11 \mathrm{G} 05$; secondary $11 \mathrm{G} 07,14 \mathrm{H} 52,14 \mathrm{H} 25$.

Keywords: elliptic curves, genus-1 curves, minimisation, reduction, descent. 


\section{Genus-1 models}

A genus-1 model (of degree 5) is a $5 \times 5$ alternating matrix of linear forms in variables $x_{1}, \ldots, x_{5}$. We write $X_{5}(R)$ for the space of all genus-1 models with coefficients in a ring $R$. Models $\Phi$ and $\Phi^{\prime}$ are $R$-equivalent if $\Phi^{\prime}=[A, B] \Phi$ for some $A, B \in \mathrm{GL}_{5}(R)$. Here the action of $A$ is via $\Phi \mapsto A \Phi A^{T}$, and the action of $B$ is via $\left(\Phi_{i j}\left(x_{1}, \ldots, x_{5}\right)\right) \mapsto\left(\Phi_{i j}\left(x_{1}^{\prime}, \ldots, x_{5}^{\prime}\right)\right)$, where $x_{j}^{\prime}=\sum_{i=1}^{5} B_{i j} x_{i}$. The determinant of the transformation $g=[A, B]$ is $\operatorname{det} g=(\operatorname{det} A)^{2} \operatorname{det} B$.

We write $\operatorname{Pf}(\Phi)$ for the row vector $\left(p_{1}, \ldots, p_{5}\right)$, where $p_{i}$ is $(-1)^{i-1}$ times the Pfaffian of the $4 \times 4$ submatrix obtained by deleting the $i$ th row and column of $\Phi$. This choice of signs is made so that $\operatorname{Pf}(\Phi) \Phi=0$. For $A \in \mathrm{GL}_{5}(R)$, we note that $\operatorname{Pf}\left(A \Phi A^{T}\right)=\operatorname{Pf}(\Phi) \operatorname{adj} A$.

A genus-1 model $\Phi \in X_{5}(K)$ over a field $K$ is nonsingular if the subscheme $\mathscr{C}_{\Phi}=\{$ rank $\Phi \leq 2\} \subset \mathbb{P}^{4}$ defined by the $4 \times 4$ Pfaffians of $\Phi$ is a smooth curve of genus 1 . We write $K\left[X_{5}\right]$ for the polynomial ring in the fifty coefficients of a genus-1 model. A polynomial $F \in K\left[X_{5}\right]$ is an invariant of weight $k$ if $F \circ g=(\operatorname{det} g)^{k} F$ for all $g=[A, B]$ with $A, B \in \mathrm{GL}_{5}(\bar{K})$. Taking $A$ and $B$ to be scalar matrices shows that an invariant of weight $k$ is a homogeneous polynomial of degree $5 k$.

Theorem 1.1. Let $c_{4}, c_{6}, \Delta \in \mathbb{Z}\left[X_{5}\right]$ be the invariants of weights 4,6 and 12 satisfying $c_{4}^{3}-c_{6}^{2}=1728 \Delta$ and scaled as specified in [Fisher 2008].

(i) A model $\Phi \in X_{5}(K)$ is nonsingular if and only if $\Delta(\Phi) \neq 0$.

(ii) There exist $a_{1}, a_{2}, a_{3}, a_{4}, a_{6} \in \mathbb{Z}\left[X_{5}\right]$ and $b_{2}, b_{4}, b_{6} \in \mathbb{Z}\left[X_{5}\right]$ satisfying

$$
\begin{aligned}
& b_{2}=a_{1}^{2}+4 a_{2}, \quad b_{4}=a_{1} a_{3}+2 a_{4}, \quad b_{6}=a_{3}^{2}+4 a_{6}, \\
& c_{4}=b_{2}^{2}-24 b_{4} \quad \text { and } \quad c_{6}=-b_{2}^{3}+36 b_{2} b_{4}-216 b_{6} .
\end{aligned}
$$

(iii) If $\Phi \in X_{5}(K)$ is nonsingular, then $\mathscr{C}_{\Phi}$ has Jacobian elliptic curve

$$
y^{2}+a_{1} x y+a_{3} y=x^{3}+a_{2} x^{2}+a_{4} x+a_{6},
$$

where $a_{i}=a_{i}(\Phi)$.

For the proof of Theorem 1.1(ii), we use the following lemma:

Lemma 1.2. Let $c_{4}, c_{6}, \Delta \in R=\mathbb{Z}\left[x_{1}, \ldots, x_{N}\right]$ be primitive polynomials satisfying $c_{4}^{3}-c_{6}^{2}=1728 \Delta$. If there exists $a_{1} \in R$ satisfying $a_{1}^{2} c_{4}+c_{6} \equiv 0(\bmod 4)$, then there exist $a_{2}, a_{3}, a_{4}, a_{6}, b_{2}, b_{4}, b_{6} \in R$ satisfying (1).

Proof. By unique factorisation in $\mathbb{F}_{3}\left[x_{1}, \ldots, x_{N}\right]$ and the Chinese remainder theorem, there exists some $b_{2} \in R$ such that $c_{4} \equiv b_{2}^{2}(\bmod 3), c_{6} \equiv-b_{2}^{3}(\bmod 3)$ and $b_{2} \equiv a_{1}^{2}(\bmod 4)$. Then $b_{2} c_{4}+c_{6} \equiv 0(\bmod 12)$, and $c_{4}^{3} \equiv c_{6}^{2} \equiv b_{2}^{2} c_{4}^{2}(\bmod 24)$. Since $c_{4}$ is primitive, it follows that $c_{4} \equiv b_{2}^{2}(\bmod 24)$. Next, putting $x=b_{2}$ in an 
identity of Kraus [1989],

$$
\left(x^{2}-c_{4}\right)^{3}=\left(x^{3}-3 x c_{4}-2 c_{6}\right)\left(x^{3}+2 c_{6}\right)+3\left(x c_{4}+c_{6}\right)^{2}+c_{6}^{2}-c_{4}^{3},
$$

we deduce $b_{2}^{3}-3 b_{2} c_{4}-2 c_{6} \equiv 0(\bmod 432)$. We put $b_{4}=\left(b_{2}^{2}-c_{4}\right) / 24$ and $b_{6}=\left(b_{2}^{3}-3 b_{2} c_{4}-2 c_{6}\right) / 432$. Then $0 \equiv c_{4}^{3}-c_{6}^{2} \equiv 16 b_{2}^{2}\left(b_{2} b_{6}-b_{4}^{2}\right)(\bmod 64)$, and so $b_{2} b_{6} \equiv b_{4}^{2}(\bmod 4)$. By unique factorisation in $\mathbb{F}_{2}\left[x_{1}, \ldots, x_{N}\right]$, there exists $a_{3} \in R$ with $b_{4} \equiv a_{1} a_{3}(\bmod 2)$. Then $b_{4}^{2} \equiv a_{1}^{2} a_{3}^{2}(\bmod 4)$, and $b_{6} \equiv a_{3}^{2}(\bmod 4)$. We put $a_{2}=\left(b_{2}-a_{1}^{2}\right) / 4, a_{4}=\left(b_{4}-a_{1} a_{3}\right) / 2$ and $a_{6}=\left(b_{6}-a_{3}^{2}\right) / 4$.

Proof of Theorem 1.1. (i) This is [Fisher 2008, Theorem 4.4(ii)].

(ii) By Lemma 1.2, it suffices to construct $a_{1} \in \mathbb{Z}\left[X_{5}\right]$ with $a_{1}^{2} c_{4}+c_{6} \equiv 0(\bmod 4)$. In [Fisher 2008, Section 10], we constructed an invariant $a_{1} \in \mathbb{F}_{2}\left[X_{5}\right]$ of weight 1 and showed that together with $\Delta$ it generates the ring of invariants in characteristic 2 . In particular, $c_{4} \equiv a_{1}^{4}(\bmod 2)$, and $c_{6} \equiv a_{1}^{6}(\bmod 2)$. So if we lift $a_{1}$ to $\mathbb{Z}\left[X_{5}\right]$, then $a_{1}^{2} c_{4}+c_{6}=2 f$ for some $f \in \mathbb{Z}\left[X_{5}\right]$. Since $a_{1}$ is an invariant $\bmod 2, a_{1}^{2}$ is an invariant $\bmod 4$ and $f$ is an invariant $\bmod 2$. Therefore, $f \equiv \lambda a_{1}^{6}(\bmod 2)$ for some $\lambda \in\{0,1\}$. Hence, $a_{1}^{2} c_{4} \pm c_{6} \equiv 0(\bmod 4)$. Specialising to one of the Weierstrass models in [Fisher 2008, Section 6] shows that the sign is + .

(iii) It is shown in [Fisher 2008, Theorem 4.4(iii)] that if $K$ is a perfect field with characteristic not 2 or 3 , then $\mathscr{C}_{\Phi}$ has Jacobian $y^{2}=x^{3}-27 c_{4}(\Phi) x-54 c_{6}(\Phi)$. The proof is now identical to that of [Cremona et al. 2010, Theorem 2.10]. This generalises a result of Artin, Rodriguez-Villegas and Tate [Artin et al. 2005] in the case $n=3$.

\section{Minimisation theorems}

Let $K$ be a discrete valuation field with ring of integers $\mathrm{O}_{K}$ and normalised valuation $v: K^{\times} \rightarrow \mathbb{Z}$. We assume throughout that the residue field $k$ is perfect. A genus- 1 model $\Phi \in X_{5}(K)$ is integral if it has coefficients in $\mathscr{O}_{K}$. If $\Phi$ is nonsingular and integral, then by Theorem 1.1 and the standard formulae for transforming Weierstrass equations, we have $v(\Delta(\Phi))=v\left(\Delta_{E}\right)+12 \ell(\Phi)$, where $\Delta_{E}$ is the minimal discriminant of $E=\operatorname{Jac}\left(\mathscr{C}_{\Phi}\right)$ and $\ell(\Phi)$ is a nonnegative integer we call the level. We say that $\Phi$ is minimal if $v(\Delta(\Phi))$, or equivalently the level, is minimal among all integral models $K$-equivalent to $\Phi$. Notice that if $\Phi^{\prime}=g \Phi$ for some $g=[A, B]$ with $A, B \in \mathrm{GL}_{5}(K)$, then $\ell\left(\Phi^{\prime}\right)=\ell(\Phi)+v(\operatorname{det} g)$.

Theorem 2.1. Let $\Phi \in X_{5}(K)$ be nonsingular.

(i) (Weak minimisation theorem) If $\mathscr{b}_{\Phi}(K) \neq \varnothing$, then $\Phi$ is $K$-equivalent to an integral model of level 0.

(ii) (Strong minimisation theorem) If $\mathfrak{b}_{\Phi}(L) \neq \varnothing$, where $L$ is an unramified extension of $K$, then $\Phi$ is $K$-equivalent to an integral model of level 0. 
In this section, we prove the weak minimisation theorem. In Section 3, we describe an explicit algorithm for minimising. Inspection of this algorithm shows that the minimal level is unchanged by an unramified extension. Theorem 2.1(ii) then follows from Theorem 2.1(i). In Section 7, we prove a converse to the strong minimisation theorem thereby showing this result is best possible.

We refer to [Cremona et al. 2010, Section 2] for notation and results analogous to those in Section 1 for genus-1 models of degree 4, i.e., quadric intersections. Let $E$ be an elliptic curve over $K$ and $D$ a $K$-rational divisor on $E$ of degree $n=4$ or 5. The complete linear system $|D|$ defines an embedding $E \subset \mathbb{P}^{n-1}$. The image is defined by a genus-1 model $\Phi \in X_{n}(K)$, and this model is uniquely determined, up to $K$-equivalence, by the pair $(E,[D])$. Moreover, every nonsingular model $\Phi \in X_{n}(K)$ with $\mathscr{C}_{\Phi}(K) \neq \varnothing$ arises in this way. Therefore, Theorem 2.1(i) is an immediate consequence of the following:

Theorem 2.2. Let $E / K$ be an elliptic curve with integral Weierstrass equation

$$
y^{2}+a_{1} x y+a_{3} y=x^{3}+a_{2} x^{2}+a_{4} x+a_{6},
$$

and let $D \in \operatorname{Div}_{K}(E)$ be a divisor on $E$ of degree $n=4$ or 5 . Then $(E,[D])$ can be represented by an integral genus-1 model with the same discriminant as (2).

The case $n=4$ is proved in [Cremona et al. 2010, Theorem 3.8]. To deduce the case $n=5$ from the case $n=4$, we use the following lemma:

Lemma 2.3. Let $D \in \operatorname{Div}_{K}(E)$ be a divisor of degree 4, and let $P \in E(K)$. Let $\ell_{i}, \alpha_{i}$ and $\beta_{i}$ for $i=1,2,3$ be linear forms in $x_{1}, \ldots, x_{4}$ over $K$. The following statements are equivalent:

(i) The pair $(E,[D])$ is represented by the quadric intersection

$$
\ell_{1} \alpha_{1}+\ell_{2} \alpha_{2}+\ell_{3} \alpha_{3}=0 \text { and } \ell_{1} \beta_{1}+\ell_{2} \beta_{2}+\ell_{3} \beta_{3}=0,
$$

and $P$ is the point defined by $\ell_{1}=\ell_{2}=\ell_{3}=0$.

(ii) The pair $(E,[D+P])$ is represented by the genus-1 model of degree 5

$$
\left(\begin{array}{ccccc}
0 & \gamma & \alpha_{1} & \alpha_{2} & \alpha_{3} \\
& 0 & \beta_{1} & \beta_{2} & \beta_{3} \\
& & 0 & \ell_{3} & -\ell_{2} \\
& - & & 0 & \ell_{1} \\
& & & & 0
\end{array}\right),
$$

where $\gamma=x_{5}$ and $P$ is the point $\left(x_{1}: \cdots: x_{5}\right)=(0: \cdots: 0: 1)$.

Proof. An isomorphism $\psi: C_{4} \rightarrow C_{5}$ between the curves $C_{4}$ and $C_{5}$ defined by (3) and (4) is given by

$$
\psi:\left(x_{1}: x_{2}: x_{3}: x_{4}\right) \mapsto\left(x_{1} \ell_{i}: x_{2} \ell_{i}: x_{3} \ell_{i}: x_{4} \ell_{i}: \alpha_{j} \beta_{k}-\alpha_{k} \beta_{j}\right)
$$


(where $i, j$ and $k$ are any cyclic permutation of 1,2 and 3 ) with inverse

$$
\psi^{-1}:\left(x_{1}: x_{2}: x_{3}: x_{4}: x_{5}\right) \mapsto\left(x_{1}: x_{2}: x_{3}: x_{4}\right) .
$$

The points $\left\{\ell_{1}=\ell_{2}=\ell_{3}=0\right\} \in C_{4}(K)$ and $(0: \cdots: 0: 1) \in C_{5}(K)$ are identified by this isomorphism. To prove the equivalence of (i) and (ii), we note that if $C_{4} \subset \mathbb{P}^{3}$ meets some plane in the divisor $D=P_{1}+P_{2}+P_{3}+P_{4}$, then the points $\psi\left(P_{i}\right)$ and $(0: \cdots: 0: 1)$ are a hyperplane section for $C_{5} \subset \mathbb{P}^{4}$.

Lemma 2.4. The genus-1 models (3) and (4) have the same invariants.

Proof. Let $\Phi$ be the matrix (4), and write $P=\operatorname{Pf}(\Phi)=\left(p_{1}, \ldots, p_{5}\right)$. Then (3) and (4) define curves $C_{4}=\left\{p_{1}=p_{2}=0\right\} \subset \mathbb{P}^{3}$ and $C_{5}=\{\operatorname{rank} \Phi \leq 2\} \subset \mathbb{P}^{4}$. According to [Fisher 2008, Section 5.4], there are invariant differentials $\omega_{4}$ on $C_{4}$ and $\omega_{5}$ on $C_{5}$ given by

$$
\omega_{n}=\frac{x_{1}^{2} d\left(x_{2} / x_{1}\right)}{\Omega_{n}\left(x_{1}, \ldots, x_{n}\right)}
$$

where

$$
\Omega_{4}=\frac{\partial p_{1}}{\partial x_{3}} \frac{\partial p_{2}}{\partial x_{4}}-\frac{\partial p_{1}}{\partial x_{4}} \frac{\partial p_{2}}{\partial x_{3}} \quad \text { and } \quad \Omega_{5}=\frac{\partial P}{\partial x_{3}} \frac{\partial \Phi}{\partial x_{5}} \frac{\partial P^{T}}{\partial x_{4}} .
$$

In the expression for $\Omega_{5}$, we have written the partial derivative of a matrix as a shorthand for the matrix of partial derivatives. Since the only entries of $\Phi$ to involve $x_{5}$ are in the top left $2 \times 2$ submatrix, it is clear that $\Omega_{4}= \pm \Omega_{5}$. Hence, the isomorphism $\psi: C_{4} \rightarrow C_{5}$ identifies the invariant differentials $\omega_{4}$ and $\omega_{5}$ (up to sign). It follows by [Fisher 2008, Proposition 5.23] that (3) and (4) have the same invariants $c_{4}, c_{6}$ and $\Delta$.

Proof of Theorem 2.2. Let $D \in \operatorname{Div}_{K}(E)$ be a divisor of degree 4, and let $P \in E(K)$. We show that if the theorem holds for $D$, then it holds for $D+P$. Suppose $(E,[D])$ is represented by an integral quadric intersection with discriminant $\Delta$. Since $\mathrm{O}_{K}$ is a principal ideal domain, $\mathrm{SL}_{4}\left(\mathscr{O}_{K}\right)$ acts transitively on $\mathbb{P}^{3}(K)$. So we may assume $P$ is the point $\left(x_{1}: x_{2}: x_{3}: x_{4}\right)=(0: 0: 0: 1)$. Our model is now of the form (3) with $\ell_{i}=x_{i}$ for $i=1,2,3$. We may choose the linear forms $\alpha_{i}$ and $\beta_{i}$ to have coefficients in $\mathscr{O}_{K}$. Then the genus- 1 model (4) is an integral model of discriminant $\Delta$ representing the pair $(E,[D+P])$.

\section{Minimisation algorithms}

For $\Phi \in X_{5}\left(\mathscr{O}_{K}\right)$, we write $\phi \in X_{5}(k)$ for its reduction mod $\pi$. The singular locus Sing $\mathscr{C}_{\phi}$ is the set of points $P \in \mathscr{C}_{\phi}$ with tangent space of dimension greater than 1 . (We make this definition regardless of whether $\mathscr{C}_{\phi}$ is a curve. In particular, all points on components of dimension at least 2 are singular.) For example, if $\phi$ takes the form (4) with $\gamma=x_{5}$ and $\ell_{i}, \alpha_{i}$ and $\beta_{i}$ linear forms in $x_{1}, \ldots, x_{4}$, then 
$P=(0: \cdots: 0: 1)$ is singular if and only if $\ell_{1}, \ell_{2}$ and $\ell_{3}$ are linearly dependent. An integral genus-1 model $\Phi \in X_{5}\left(\mathscr{O}_{K}\right)$ is saturated if its $4 \times 4$ Pfaffians $p_{1}, \ldots, p_{5}$ are linearly independent $\bmod \pi$. We write $I_{m}$ for the $m \times m$ identity matrix.

Our algorithm for minimising genus- 1 models of degree 5 generalises the algorithm for models of degree 3 in [Cremona et al. 2010, Section 4B].

Theorem 3.1. Let $\Phi \in X_{5}\left(\mathscr{O}_{K}\right)$ be saturated and of positive level.

(i) The singular locus $\operatorname{Sing} \mathscr{C}_{\phi}$ does not span $\mathbb{P}^{4}$.

(ii) Let $B \in \mathrm{GL}_{5}\left(\mathrm{O}_{K}\right)$ represent a change of coordinates on $\mathbb{P}^{4}$ mapping the linear span of the singular locus in (i) to $\left\{x_{m+1}=\cdots=x_{5}=0\right\}$. Then there exist $A \in \mathrm{GL}_{5}(K)$ and $\mu \in K^{\times}$such that $\left[A, \mu \operatorname{Diag}\left(I_{m}, \pi I_{5-m}\right) B\right] \Phi$ is an integral model of the same or smaller level.

(iii) If $\Phi$ is nonminimal, then repeating the procedure in (ii) either gives a nonsaturated model or decreases the level after finitely many iterations.

As it stands, Theorem 3.1 does not give an algorithm for minimising since we must show how to find $A$ and $\mu$ in (ii) and show how to decrease the level of a nonsaturated model. We do this in Theorem 3.2 below. Theorem 3.1 is proved in Sections 4 and 5. In Section 6, we bound the number of iterations required in (iii).

Theorem 3.2. Let $\Phi \in X_{5}\left(\mathscr{O}_{K}\right)$ be nonsingular. Let $\ell_{0}$ be the minimum of the levels of all integral models that are $K$-equivalent to $\Phi$ via a transformation of the form $\left[A, \mu I_{5}\right]$, where $A \in \mathrm{GL}_{5}(K)$ and $\mu \in K^{\times}$.

(i) We may compute an integral model of the form $\left[A, \mu I_{5}\right] \Phi$ with level $\ell_{0}$ as follows:

Step 1. Write $\operatorname{Pf}(\Phi)=\left(p_{1}, \ldots, p_{5}\right)$. Compute $A=\left(a_{i j}\right) \in \mathrm{GL}_{5}(K)$ and quadrics $q_{1}, \ldots, q_{5} \in \mathrm{O}_{K}\left[x_{1}, \ldots, x_{5}\right]$ such that $p_{j}=\sum_{i=1}^{5} a_{i j} q_{i}$ and $q_{1}, \ldots, q_{5}$ are linearly independent modulo $\pi$. Then replace $\Phi$ by $\left[A, \mu I_{5}\right] \Phi$, where $\mu \in K^{\times}$is chosen so that $\Phi$ has coefficients in $\mathbb{O}_{K}$ not all in $\pi \mathrm{O}_{K}$.

Step 2. Replace $\Phi$ by $\left[A, I_{5}\right] \Phi$, where $A \in \mathrm{GL}_{5}\left(\mathbb{O}_{K}\right)$ is chosen so that the first two rows of $\Phi$ are divisible by $\pi^{e}$ with $e \geq 0$ as large as possible. Then divide the first row and column by $\pi^{e}$.

(ii) If the model computed in Step 1 is nonsaturated, then we may compute an integral model of level smaller than $\ell_{0}$ by modifying Step 2 so that we divide the first two rows and columns by $\pi^{e}$ and then make a transformation of the form $\left[I_{5}, B\right]$ to preserve integrality.

Proof. With the notation of Step 1, we have

$\operatorname{Pf}\left(A \Phi A^{T}\right)=\operatorname{Pf}(\Phi) \operatorname{adj} A=\left(q_{1}, \ldots, q_{5}\right) A \operatorname{adj} A=(\operatorname{det} A)\left(q_{1}, \ldots, q_{5}\right)$. 
So after Step 1, we have $\operatorname{Pf}(\Phi)=\left(\lambda q_{1}, \ldots, \lambda q_{5}\right)$, where $\lambda=\mu^{2} \operatorname{det} A \in \mathcal{O}_{K}$. We split into the cases $v(\lambda)=0$ and $v(\lambda) \geq 1$. First we need two lemmas.

Lemma 3.3. Let $\Phi, \Phi^{\prime} \in X_{5}\left(\mathscr{O}_{K}\right)$ be nonsingular models with $\Phi^{\prime}=\left[A, \mu I_{5}\right] \Phi$ for some $A \in \mathrm{GL}_{5}(K)$ and $\mu \in K^{\times}$.

(i) If $\Phi$ is saturated, then $\ell\left(\Phi^{\prime}\right) \geq \ell(\Phi)$ with equality if and only if $\Phi$ and $\Phi^{\prime}$ are $\mathrm{O}_{K}$-equivalent.

(ii) If $\Phi$ and $\Phi^{\prime}$ are of the form output by Step 1 , then they are $\mathscr{O}_{K}$-equivalent. Proof. We have $\operatorname{Pf}\left(\Phi^{\prime}\right)=\operatorname{Pf}(\Phi) M$, where $M=\mu^{2}$ adj $A$.

(i) Since $\Phi$ is saturated, $M$ has entries in $0_{K}$. Hence, $\ell\left(\Phi^{\prime}\right)-\ell(\Phi)=\frac{1}{2} v(\operatorname{det} M) \geq 0$ with equality if and only if $M \in \mathrm{GL}_{5}\left(\mathrm{O}_{K}\right)$. If $M \in \mathrm{GL}_{5}\left(\mathrm{O}_{K}\right)$, then replacing [ $\left.A, \mu I_{5}\right]$ by $\left[\lambda A, \lambda^{-2} \mu I_{5}\right]$ for suitable $\lambda \in K^{\times}$, we may assume $A \in \mathrm{GL}_{5}\left(\mathscr{O}_{K}\right)$. Since $\Phi$ and $\Phi^{\prime}$ have the same level, they must therefore be $\mathscr{O}_{K}$-equivalent.

(ii) Since $\operatorname{Pf}(\Phi)$ and $\operatorname{Pf}\left(\Phi^{\prime}\right)$ are scalar multiples of bases for the same $\mathscr{O}_{K}$-module, some scalar multiple of $M$ belongs to $\mathrm{GL}_{5}\left(O_{K}\right)$. So after replacing $\left[A, \mu I_{5}\right]$ by $\left[\lambda A, \lambda^{-2} \mu I_{5}\right]$ for suitable $\lambda \in K^{\times}$, we may assume $A \in \mathrm{GL}_{5}\left(\mathcal{O}_{K}\right)$. Since $\Phi$ and $\Phi^{\prime}$ are primitive, they must therefore be $\mathrm{O}_{K}$-equivalent.

Lemma 3.4. Let $\phi \in X_{5}(k)$ be a genus-1 model all of whose $4 \times 4$ Pfaffians are identically zero. Then $\phi$ is $k$-equivalent to either

$$
\left(\begin{array}{ccccc}
0 & \ell_{2} & \ell_{3} & \ell_{4} & \ell_{5} \\
& 0 & 0 & 0 & 0 \\
& & 0 & 0 & 0 \\
& & & 0 & 0 \\
& & & & \\
& & & & 0
\end{array}\right) \quad \text { or } \quad\left(\begin{array}{ccccc}
0 & x_{1} & x_{2} & 0 & 0 \\
& 0 & x_{3} & 0 & 0 \\
& & 0 & 0 & 0 \\
& - & & 0 & 0 \\
& & & & 0
\end{array}\right)
$$

where $\ell_{2}, \ldots, \ell_{5}$ are linear forms.

We now complete the proof of Theorem 3.2. Let $e=v(\lambda)$. If $e=0$, then $\Phi$ is saturated and we are done by Lemma 3.3(i). So suppose $e \geq 1$. In Step 1, the matrix $A$ has entries in $\mathscr{O}_{K}$. So $v(\mu) \leq 0$, and the level is increased by

$$
2 v(\operatorname{det} A)+5 v(\mu) \leq 2 v\left(\mu^{2} \operatorname{det} A\right)=2 e .
$$

Lemma 3.3(ii) shows that when we apply Step 1 to both $\Phi$ and the model implicit in the definition of $\ell_{0}$, we obtain models that are $O_{K}$-equivalent. So it will suffice to show that Step 2 reduces the level by $2 e$, whereas the modified version in (ii) reduces the level by more than $2 e$.

Since $\operatorname{Pf}(\Phi)=\left(\lambda q_{1}, \ldots, \lambda q_{5}\right)$, we have $\left(q_{1}, \ldots, q_{5}\right) \Phi=0$. The reduction of $\Phi$ takes one of the forms specified in Lemma 3.4. In the first case, we have $q_{1} \ell_{j} \equiv 0(\bmod \pi)$ for $j=2, \ldots, 5$. This contradicts the choices of $q_{1}, \ldots, q_{5}$ and $\mu$ in Step 1. So we must be in the second case. Replacing $\Phi$ by an $\mathscr{O}_{K}$-equivalent 
model, we may assume it takes the form (4) with $\ell_{i}=x_{i}$ for $i=1,2,3$ and $\alpha_{1}$, $\alpha_{2}, \alpha_{3}, \beta_{1}, \beta_{2}, \beta_{3}$ and $\gamma$ linear forms that vanish $\bmod \pi$. By row and column operations, we may assume $\alpha_{2} \in\left\langle x_{2}, \ldots, x_{5}\right\rangle$ and $\alpha_{3} \in\left\langle x_{3}, \ldots, x_{5}\right\rangle$. Then since $\pi^{e} \mid\left(x_{1} \alpha_{1}+x_{2} \alpha_{2}+x_{3} \alpha_{3}\right)$, we have $\pi^{e} \mid \alpha_{1}, \alpha_{2}, \alpha_{3}$. Likewise, we may assume $\pi^{e} \mid \beta_{1}, \beta_{2}, \beta_{3}$. The remaining Pfaffians show that $\pi^{e} \mid \gamma$. Step 2 and its modified version in (ii) now reduce the level by $2 e$ and $3 e$, respectively.

Corollary 3.5. For the proof of Theorem 3.1, we are free to replace $\Phi$ by an $\mathrm{O}_{K}$-equivalent model and to replace $K$ by an unramified field extension.

Proof. Let $\Phi_{1}, \Phi_{2} \in X_{5}\left(\mathscr{O}_{K}\right)$ be $\mathscr{O}_{K}$-equivalent models and $\Phi_{1}^{\prime}, \Phi_{2}^{\prime} \in X_{5}\left(\mathscr{O}_{K}\right)$ the models returned by Theorem 3.1(ii). Lemma 3.3(i) and [Cremona et al. 2010, Lemma 4.1] together show that if $\Phi_{1}^{\prime}$ is saturated and $\ell\left(\Phi_{1}^{\prime}\right)=\ell\left(\Phi_{2}^{\prime}\right)$, then $\Phi_{1}^{\prime}$ and $\Phi_{2}^{\prime}$ are $\mathrm{O}_{K}$-equivalent. Therefore, the number of iterations required in Theorem 3.1(iii) depends only on the $\hat{O}_{K}$-equivalence class of $\Phi$.

For the final statement, we note that the performance of the algorithms in Theorems 3.1 and 3.2 is unchanged by an unramified field extension.

Replacing $K$ by its strict Henselisation, we may assume in the next three sections that $K$ is Henselian and its residue field $k$ is algebraically closed.

\section{The singular locus}

In this section and the next, we prove Theorem 3.1.

Lemma 4.1. Let $\phi \in X_{5}(k)$ be a genus-1 model. Suppose $\Gamma \subset \mathscr{C}_{\phi}$ is either a line or a (nonsingular) conic. Then either $\Gamma \subset \operatorname{Sing} \mathscr{C}_{\phi}$ or

$$
\#\left(\Gamma \cap \operatorname{Sing} \mathscr{C}_{\phi}\right)= \begin{cases}1 & \text { if } c_{4}(\phi)=c_{6}(\phi)=0, \\ 2 & \text { otherwise }\end{cases}
$$

Proof. (i) If $\mathscr{C}_{\phi}$ contains the line $\Gamma=\left\{x_{3}=x_{4}=x_{5}=0\right\}$ but not every point on $\Gamma$ is singular, then (unless $\mathscr{C}_{\phi}$ is a cone, which is an easy special case with $c_{4}(\phi)=c_{6}(\phi)=0$ ) we may suppose $\phi$ is

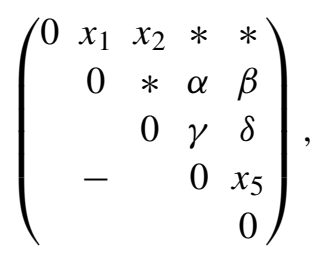

where $\alpha, \beta, \gamma, \delta$ and the entries $*$ are linear forms in $x_{3}, x_{4}, x_{5}$. By row and column operations (and substitutions for $x_{1}$ and $x_{2}$ ), we may suppose $\alpha, \beta, \gamma$ and $\delta$ do not 
involve $x_{5}$. We write $\alpha=\alpha_{3} x_{3}+\alpha_{4} x_{4}, \ldots, \delta=\delta_{3} x_{3}+\delta_{4} x_{4}$ and put

$$
q(s, t)=\operatorname{det}\left(\left(\begin{array}{ll}
\gamma_{3} & \gamma_{4} \\
\delta_{3} & \delta_{4}
\end{array}\right) s-\left(\begin{array}{cc}
\alpha_{3} & \alpha_{4} \\
\beta_{3} & \beta_{4}
\end{array}\right) t\right)
$$

By the Jacobian criterion, we have

$$
\Gamma \cap \operatorname{Sing} \mathscr{C}_{\phi}=\{(s: t: 0: 0: 0) \mid q(s, t)=0\} .
$$

A calculation using Lemma 2.4 shows that $c_{4}(\phi)=\Delta(q)^{2}$ and $c_{6}(\phi)=-\Delta(q)^{3}$, where $\Delta(q)$ is the discriminant of the binary quadratic form $q$.

(ii) Suppose $\mathscr{C}_{\phi}$ contains the conic $\Gamma=\left\{f\left(x_{1}, x_{2}, x_{3}\right)=x_{4}=x_{5}=0\right\}$ but not every point on $\Gamma$ is singular. Let $\operatorname{Pf}(\phi)=\left(p_{1}, \ldots, p_{5}\right)$. Replacing $\phi$ by an equivalent model, we may suppose $p_{i}\left(x_{1}, x_{2}, x_{3}, 0,0\right)=0$ for $i=1,2,3,4$ and $p_{5}\left(x_{1}, x_{2}, x_{3}, 0,0\right)=f$. Since $\operatorname{Pf}(\phi) \phi=0$ and $\Gamma$ is not contained in any component of $\mathscr{C}_{\phi}$ of higher dimension, we may further suppose the last column of $\phi$ has entries $x_{4}, x_{5}, 0,0,0$. The monomials appearing in the invariants $c_{4}$ and $c_{6}$ are limited by the fact they are invariant under all pairs of diagonal matrices. These restrictions show that $c_{4}(\phi)$ and $c_{6}(\phi)$ are unchanged if we set $x_{4}=x_{5}=0$ in all entries of $\phi$ outside the last row and column. Writing $f=\sum_{i \leq j} a_{i j} x_{i} x_{j}$ and $\phi_{34}=\sum b_{i} x_{i}$, we put

$$
\delta=\left|\begin{array}{cccc}
2 a_{11} & a_{12} & a_{13} & b_{1} \\
a_{12} & 2 a_{22} & a_{23} & b_{2} \\
a_{13} & a_{23} & 2 a_{33} & b_{3} \\
b_{1} & b_{2} & b_{3} & 0
\end{array}\right| .
$$

A calculation using Lemma 2.4 shows that $c_{4}(\phi)=\delta^{2}$ and $c_{6}(\phi)=-\delta^{3}$. By a change of coordinates, we may suppose $f=x_{1} x_{3}-x_{2}^{2}$. Then $\delta$ is the discriminant of the binary quadratic form $q(s, t)=\phi_{34}\left(s^{2}, s t, t^{2}, 0,0\right)$, and by the Jacobian criterion,

$$
\Gamma \cap \operatorname{Sing} \mathscr{C}_{\phi}=\left\{\left(s^{2}: s t: t^{2}: 0: 0\right) \mid q(s, t)=0\right\} .
$$

Lemma 4.2. Let $\phi \in X_{5}(k)$ be a genus-1 model. Suppose the $4 \times 4$ Pfaffians $p_{1}, \ldots, p_{5}$ are linearly independent and $c_{4}(\phi)=c_{6}(\phi)=0$. Then either $\operatorname{Sing} \mathscr{C}_{\phi}$ is a linear subspace of $\mathbb{P}^{4}$ or $\phi$ is equivalent to a model of the form

$$
\left(\begin{array}{ccccc}
0 & \xi & \alpha & \beta & \eta \\
& 0 & \gamma & \delta & x_{5} \\
& & 0 & x_{5} & 0 \\
& - & & 0 & 0 \\
& & & & 0
\end{array}\right)
$$

where $\xi, \eta, \alpha, \beta, \gamma$ and $\delta$ are linear forms in $x_{1}, \ldots, x_{5}$. 
Proof. If $P_{1}, P_{2} \in \operatorname{Sing} \mathscr{C}_{\phi}$ are distinct and the line $\ell$ between them is contained in $\mathscr{C}_{\phi}$, then by Lemma 4.1, we have $\ell \subset \operatorname{Sing} \mathscr{C}_{\phi}$. So either Sing $\mathscr{C}_{\phi}$ is a linear subspace of $\mathbb{P}^{4}$ or there exist $P_{1}, P_{2} \in \operatorname{Sing} \mathscr{C}_{\phi}$ joined by a line not contained in $\mathscr{C}_{\phi}$. We move these points to $(1: 0: \cdots: 0)$ and $(0: 1: \cdots: 0)$. Writing $\phi=\sum x_{i} M_{i}$, the matrices $M_{1}$ and $M_{2}$ have rank 2, but their sum has rank 4. Therefore, $\phi$ is equivalent to a model with $\phi_{12}=x_{1}, \phi_{34}=x_{2}$ and all other $\phi_{i j}$ (for $i<j$ ) linear forms in $x_{3}, x_{4}, x_{5}$. Since $P_{1}$ and $P_{2}$ are singular, $\phi_{35}$ and $\phi_{45}$ are linearly dependent and $\phi_{15}$ and $\phi_{25}$ are linearly dependent. So the space of linear forms spanned by the entries of the last column has dimension at most 2. In fact, it has dimension exactly 2 since $p_{1}, \ldots, p_{5}$ are linearly independent.

Replacing $\phi$ by an equivalent model, we may assume it has last column with entries $x_{4}, x_{5}, 0,0,0$. The transformation used here does not move $P_{1}$ and $P_{2}$ but may change the matrices $M_{1}$ and $M_{2}$. Let $\Gamma=\left\{x_{4}=x_{5}=p_{5}=0\right\} \subset \mathscr{b}_{\phi}$. Then $P_{1}$ and $P_{2}$ are contained in $\Gamma$, but the line between them is not. It follows that $\Gamma$ is either a nonsingular conic or a pair of concurrent lines. In either case, Lemma 4.1 shows that $\Gamma \subset \operatorname{Sing} \mathscr{C}_{\phi}$. By the Jacobian criterion, it follows that $\phi_{34} \in\left\langle x_{4}, x_{5}\right\rangle$. However, $\phi_{34}$ is nonzero since $p_{1}, \ldots, p_{5}$ are linearly independent. Therefore, $\phi$ is equivalent to a model of the form (5).

Lemma 4.3. Let $\Phi \in X_{5}\left(\mathscr{O}_{K}\right)$ be a saturated nonsingular model with reduction $\phi$ of the form (5). Suppose $\operatorname{Sing} \mathscr{C}_{\phi}$ has linear span $\left\{x_{m+1}=\cdots=x_{5}=0\right\}$.

(i) There exist $A \in \mathrm{GL}_{5}(K)$ and $\mu \in K^{\times}$such that $\left[A, \mu \operatorname{Diag}\left(I_{m}, \pi I_{5-m}\right)\right] \Phi$ is an integral model of the same or smaller level.

(ii) Suppose that either $\delta=0$ and $\Phi_{45} \equiv 0\left(\bmod \pi^{2}\right)$ or $\Phi_{35} \equiv \Phi_{45} \equiv 0\left(\bmod \pi^{2}\right)$. Then there is a transformation as in (i) that decreases the level.

Proof. Computing the $4 \times 4$ Pfaffians of (5), we find

$$
\mathscr{C}_{\phi}=\left\{\eta=x_{5}=\alpha \delta-\beta \gamma=0\right\} \cup\left\{\gamma=\delta=x_{5}=0\right\} .
$$

First suppose $\gamma, \delta$ and $x_{5}$ are linearly dependent. By an $\mathscr{O}_{K}$-equivalence, we may assume $\delta=0$. Then $\left\{\gamma=x_{5}=0\right\} \subset \operatorname{Sing} \mathscr{C}_{\phi} \subset\left\{x_{5}=0\right\}$. Therefore, $m=3$ or 4 . The required transformations are as follows:

\begin{tabular}{r|c|c} 
& $m=3$ & $m=4$ \\
\hline (i) & $A=\operatorname{Diag}(\pi, 1,1,1,1), \mu=\pi^{-1}$ & $A=\operatorname{Diag}(\pi, \pi, 1,1,1), \mu=\pi^{-1}$ \\
(ii) & $A=\operatorname{Diag}(\pi, 1,1,1,1), \quad \mu=\pi^{-1}$ & $A=\operatorname{Diag}\left(\pi, 1,1, \pi^{-1}, \pi^{-1}\right), \mu=1$
\end{tabular}

Now suppose $\gamma, \delta$ and $x_{5}$ are linearly independent. Since $\Phi$ is saturated, $\eta$ and $x_{5}$ are linearly independent. A calculation shows $\operatorname{Sing} \mathscr{C}_{\phi}$ is the first of the two components in (6). Therefore, $m=2$ or 3 . If $m=2$, then we may assume $\beta, \gamma, \delta, \eta$ and $\phi_{25}$ 
are linear forms in $x_{3}, x_{4}$ and $x_{5}$. The required transformations are as follows:

\begin{tabular}{r|c|c} 
& $m=2$ & $m=3$ \\
\hline (i) & $A=\operatorname{Diag}(\pi, 1,1,1,1), \mu=\pi^{-1}$ & $A=\operatorname{Diag}\left(1,1,1,1, \pi^{-1}\right), \mu=1$ \\
(ii) & $A=\operatorname{Diag}\left(1,1,1, \pi^{-1}, \pi^{-1}\right), \quad \mu=1$ & $A=\operatorname{Diag}\left(\pi, \pi, 1,1, \pi^{-1}\right), \mu=\pi^{-1}$
\end{tabular}

We now prove the first two parts of Theorem 3.1. Let $\Phi \in X_{5}\left(\mathscr{O}_{K}\right)$ be saturated and of positive level. Lemma 4.2 shows that either $\operatorname{Sing} \mathscr{C}_{\phi}$ is a linear subspace or $\mathscr{C}_{\phi}$ is contained in a hyperplane. Since $\mathscr{C}_{\phi}$ is defined by five linearly independent quadrics, it cannot be all of $\mathbb{P}^{4}$. This proves Theorem 3.1(i).

The proof of Theorem 3.1(ii) in the case $\phi$ takes the form (5) was already given in Lemma 4.3(i). So by Lemma 4.2, we may assume Sing $\mathscr{C}_{\phi}=\left\{x_{m+1}=\cdots=x_{5}=0\right\}$. We apply Lemma 3.4 to the reduction $\bmod \pi$ of $\left[I_{5}, \operatorname{Diag}\left(I_{m}, \pi I_{5-m}\right)\right] \Phi$. In the second case of that lemma, we have $m \geq 3$. We take $A=\operatorname{Diag}\left(1,1,1,1, \pi^{-1}\right)$ and $\mu=1$. Otherwise, we are in the first case. If $m \geq 2$, then we take $\mu=\pi^{-1}$ and $A=\operatorname{Diag}(\pi, 1,1,1,1)$. It remains to treat the case $m=1$; in other words, the case Sing $\mathscr{C}_{\phi}$ is a point.

By [Fisher 2008, Lemma 5.8], every component of $\mathscr{C}_{\phi}$ has dimension at least 1. So if Sing $\mathscr{C}_{\phi}$ is just a point, then there are also smooth points on $\mathscr{C}_{\phi}$. Since $K$ is Henselian, it follows that $\mathscr{C}_{\Phi}(K) \neq \varnothing$, and so by Theorem 2.1(i), $\Phi$ is nonminimal. With this extra hypothesis, we show in the next section that the singular point on $\mathscr{C}_{\phi}$ is nonregular (as a point on the $\mathfrak{O}_{K}$-scheme $\mathscr{C}_{\Phi}$ ).

We may suppose $\phi_{12}=x_{1}$ and all other $\phi_{i j}$ (for $i<j$ ) are linear forms in $x_{2}, \ldots, x_{5}$. Since $P=(1: 0: \cdots: 0)$ is singular, $\phi_{34}, \phi_{35}$ and $\phi_{45}$ are linearly dependent. So replacing $\Phi$ by an $\mathbb{O}_{K}$-equivalent model, we may assume $\phi_{45}=0$. In the presence of the stronger condition that $P$ is nonregular, we may further arrange that the coefficient of $x_{1}$ in $\Phi_{45}$ is divisible by $\pi^{2}$. Taking $A=\operatorname{Diag}\left(1,1,1, \pi^{-1}, \pi^{-1}\right)$ and $\mu=1$ now preserves the level.

\section{Weights and slopes}

In this section, we complete the proof of Theorem 3.1.

Definition 5.1. (i) The set of weights is

$$
\mathcal{W}=\left\{\begin{array}{l|c}
(r, s) \in \mathbb{Z}^{5} \times \mathbb{Z}^{5} \mid \begin{array}{c}
r_{1} \leq r_{2} \leq \cdots \leq r_{5}, s_{1} \leq s_{2} \leq \cdots \leq s_{5} \\
2 \sum_{i=1}^{5} r_{i}=1+\sum_{i=1}^{5} s_{i}
\end{array}
\end{array}\right\} .
$$

(ii) A weight for $\Phi \in X_{5}\left(\mathscr{O}_{K}\right)$ is $(r, s) \in \mathcal{W}$ such that the model

$$
\left[\operatorname{Diag}\left(\pi^{-r_{1}}, \ldots, \pi^{-r_{5}}\right), \operatorname{Diag}\left(\pi^{s_{1}}, \ldots, \pi^{s_{5}}\right)\right] \Phi
$$

has coefficients in $\mathrm{O}_{K}$. 
(iii) Let $w=(r, s)$ and $w^{\prime}=\left(r^{\prime}, s^{\prime}\right)$ be weights. Then $w$ dominates $w^{\prime}$ if

$$
\max \left(r_{i}+r_{j}-s_{k}, 0\right) \geq \max \left(r_{i}^{\prime}+r_{j}^{\prime}-s_{k}^{\prime}, 0\right)
$$

for all $1 \leq i<j \leq 5$ and $1 \leq k \leq 5$.

Let $\mathbf{1}=(1,1, \ldots, 1)$. Then $\lambda \in \mathbb{Z}$ acts on $W$ as $(r, s) \mapsto(r+\lambda \mathbf{1}, s+2 \lambda \mathbf{1})$. Since weights in the same $\mathbb{Z}$-orbit determine the same transformation (7), we may regard such weights as equivalent.

Lemma 5.2. Let $\Phi \in X_{5}\left(\mathbb{O}_{K}\right)$ be an integral genus-1 model.

(i) If $\Phi$ is nonminimal, then it is $\mathbb{O}_{K}$-equivalent to a model with a weight.

(ii) If $\Phi$ has weight $w$ and $w$ dominates $w^{\prime}$, then $\Phi$ has weight $w^{\prime}$.

Proof. (i) By hypothesis, there exist $A, B \in \mathrm{GL}_{5}(K)$ with $[A, B] \Phi$ integral and $2 v(\operatorname{det} A)+v(\operatorname{det} B)=-1$. We put $A$ and $B$ in Smith normal form.

(ii) Let $\Phi=\left(\Phi_{i j}\right)$ with $\Phi_{i j}=\sum_{k} a_{i j k} x_{k}$. Then $\Phi$ has weight $(r, s)$ if and only if $v\left(a_{i j k}\right) \geq \max \left(r_{i}+r_{j}-s_{k}, 0\right)$ for all $1 \leq i<j \leq 5$ and $1 \leq k \leq 5$.

Lemma 5.3. Let $\Phi \in X_{5}\left(\mathscr{O}_{K}\right)$ have weight $(r, s) \in \mathcal{W}$ with either $r_{1}+r_{4}>s_{1}$ or $r_{2}+r_{3}>s_{1}$. Then $P=(1: 0: \cdots: 0) \in \mathscr{C}_{\phi}$ is a singular point. Moreover, if $s_{1}<s_{3}$, then $P$ is nonregular (as a point on the $\mathbb{O}_{K}$-scheme $\mathscr{C}_{\Phi}$ ).

Proof. We write $\phi=\sum x_{i} M_{i}$. If $r_{1}+r_{4}>s_{1}$, then the only nonzero entries of $M_{1}$ are in the top left $3 \times 3$ submatrix. If $r_{2}+r_{3}>s_{1}$, then the only nonzero entries of $M_{1}$ are in the first row and column. In both cases, rank $M_{1} \leq 2$, and so $P \in \mathscr{C}_{\phi}$. If $M_{1}=0$, then $P$ is singular (and nonregular). So we may assume $M_{1} \neq 0$. We are free to multiply rows of $\Phi$ by units in $\hat{O}_{K}$ and to subtract $O_{K}$-multiples of later rows from earlier rows (it being understood that we also make the corresponding column operations). In particular, these operations do not upset our hypothesis that $\Phi$ has weight $(r, s)$. Let $E_{i j}$ be the $5 \times 5$ matrix with a 1 in the $(i, j)$-place and 0 s elsewhere. By row and column operations, we reduce to the case $M_{1}=E_{i j}-E_{j i}$, where $(i, j) \in\{(1,2),(1,3),(1,4),(1,5),(2,3)\}$. Let $a<b<c$ be chosen such that $\{i, j, a, b, c\}=\{1, \ldots, 5\}$. Since $r_{i}+r_{j} \leq s_{1} \leq s_{2}$, it follows by the definition of $W$ that

$$
s_{3}+s_{4}+s_{5}<\left(r_{a}+r_{b}\right)+\left(r_{a}+r_{c}\right)+\left(r_{b}+r_{c}\right) .
$$

Therefore, at least one of the following three inequalities holds:

$$
\begin{array}{cccc}
s_{3}<r_{a}+r_{b} & \Rightarrow & \phi_{a b}, \phi_{a c}, \phi_{b c} & \in\left\langle x_{4}, x_{5}\right\rangle, \\
s_{4}<r_{a}+r_{c} & \Rightarrow & \phi_{a c}, \phi_{b c} & \in\left\langle x_{5}\right\rangle, \\
s_{5}<r_{b}+r_{c} & \Longrightarrow & \phi_{b c} & =0 .
\end{array}
$$

Since the tangent space at $P$ is $\left\{\phi_{a b}=\phi_{a c}=\phi_{b c}=0\right\}$, it follows that $P \in \mathscr{C}_{\phi}$ is a singular point. 
If $s_{1}<s_{3}$, then the same argument shows there is some $\mathbb{O}_{K}$-linear combination of $\Phi_{a b}, \Phi_{a c}$ and $\Phi_{b c}$ (with not all coefficients in $\pi \mathscr{O}_{K}$ ) that not only vanishes mod $\pi$ but whose coefficient of $x_{1}$ vanishes mod $\pi^{2}$. Hence, $P$ is nonregular.

Lemma 5.4. Let $(r, s) \in \mathcal{W}$ be a weight with $r_{1}+r_{4} \leq s_{1}$ and $r_{2}+r_{3} \leq s_{1}$. Then $(r, s)$ dominates one of the weights $w_{1}, \ldots, w_{7}$ in the following table:

\begin{tabular}{c|ccccc|ccccc} 
& $r_{1}$ & $r_{2}$ & $r_{3}$ & $r_{4}$ & $r_{5}$ & $s_{1}$ & $s_{2}$ & $s_{3}$ & $s_{4}$ & $s_{5}$ \\
\hline$w_{1}$ & 0 & 0 & 0 & 0 & 1 & 0 & 0 & 0 & 0 & 1 \\
$w_{2}$ & 0 & 0 & 1 & 1 & 1 & 1 & 1 & 1 & 1 & 1 \\
$w_{3}$ & 0 & 0 & 1 & 1 & 2 & 1 & 1 & 1 & 2 & 2 \\
$w_{4}$ & 0 & 1 & 1 & 2 & 2 & 2 & 2 & 2 & 2 & 3 \\
$w_{5}$ & 0 & 1 & 1 & 2 & 3 & 2 & 2 & 2 & 3 & 4 \\
$w_{6}$ & 0 & 1 & 1 & 2 & 3 & 2 & 2 & 3 & 3 & 3 \\
$w_{7}$ & 0 & 1 & 2 & 3 & 4 & 3 & 3 & 4 & 4 & 5
\end{tabular}

Proof. We checked the lemma by writing a computer program using the simplex algorithm. See the proof of Lemma 6.1 for details.

Definition 5.5. The slope of $\Phi \in X_{5}\left(O_{K}\right)$ is the least possible value of $v(\operatorname{det} B)$ for $B \in \mathrm{GL}_{5}(K)$ a matrix with entries in $\mathrm{O}_{K}$ for which there exist $A \in \mathrm{GL}_{5}(K)$ and $\mu \in K^{\times}$such that $[A, \mu B] \Phi$ is an integral model of smaller level.

We now complete the proof of Theorem 3.1. Since $\Phi \in X_{5}\left(\mathscr{O}_{K}\right)$ is nonminimal, it has a slope $\sigma$, say. Lemma 3.3(i) shows that if $\sigma=0$, then $\Phi$ is nonsaturated. So we may assume $\sigma>0$. By Lemma 5.2 (and Corollary 3.5), we may replace $\Phi$ by an $\mathbb{O}_{K}$-equivalent model with a weight, say $(r, s)$. Moreover, we may assume the weight realises the slope, i.e., $\sigma=\sum_{i=1}^{5}\left(s_{i}-s_{1}\right)$.

Suppose that either $r_{1}+r_{4}>s_{1}$ or $r_{2}+r_{3}>s_{1}$. Since $\sigma>0$, there exists $1 \leq m \leq 4$ such that $s_{1}=\cdots=s_{m}<s_{m+1}$. Lemma 5.3 shows (by first making unimodular transformations involving only $x_{1}, \ldots, x_{m}$ ) that

$$
\left\{x_{m+1}=\cdots=x_{5}=0\right\} \subset \operatorname{Sing} \mathscr{C}_{\phi} .
$$

Moreover, if $m=1$, then the point we have constructed is nonregular. (This is needed to complete the proof of Theorem 3.1(ii) at the end of Section 4.)

Regardless of whether we have equality in (8), it follows that if the level is preserved, then the slope is decreased. So after finitely many iterations, $\Phi$ is either nonsaturated or has weight $(r, s)$ with $r_{1}+r_{4} \leq s_{1}$ and $r_{2}+r_{3} \leq s_{1}$. In this last case, Lemmas 5.2 and 5.4 show that $\Phi$ has weight $w$ for some $w \in\left\{w_{1}, \ldots, w_{7}\right\}$. If $w \in\left\{w_{1}, w_{2}, w_{6}\right\}$, then $\Phi$ is nonsaturated. If $w \in\left\{w_{5}, w_{7}\right\}$, then $\Phi$ is $\mathscr{O}_{K}$-equivalent to a model with weight $w_{3}$. (This is achieved by a unimodular transformation involving only the second and third rows and columns, respectively a unimodular 
transformation involving only $x_{3}$ and $x_{4}$.) Finally, if $w \in\left\{w_{3}, w_{4}\right\}$, then $\Phi$ is $\mathrm{O}_{K}$-equivalent to a model of the form considered in Lemma 4.3(ii).

\section{The number of iterations}

We have shown that if we start with a nonminimal model, then iterating the procedure in Theorem 3.1(ii) eventually gives a nonsaturated model or decreases the level. In this section, we show that the maximum number of iterations required is 5. (In our Magma implementation, we count the use of Theorem 3.2 to decrease the level of a nonsaturated model as a further iteration. With this convention, the maximum number of iterations is 6 .)

Lemma 6.1. Let $(r, s) \in \mathcal{W}$ be a weight. Then $(r, s)$ dominates one of the weights $w_{1}, \ldots, w_{29}$ in the following table. (The weights in Lemma 5.4 appear with new numberings. We have marked these weights in bold.)

\begin{tabular}{c|rrrrr|ccccc|cc|ccccc|ccccc|c} 
& $r_{1}$ & $r_{2}$ & $r_{3}$ & $r_{4}$ & $r_{5}$ & $s_{1}$ & $s_{2}$ & $s_{3}$ & $s_{4}$ & $s_{5}$ & $\lambda_{v}$ & & $r_{1}$ & $r_{2}$ & $r_{3}$ & $r_{4}$ & $r_{5}$ & $s_{1}$ & $s_{2}$ & $s_{3}$ & $s_{4}$ & $s_{5}$ & $\lambda_{v}$ \\
\hline$w_{1}$ & 0 & 0 & 0 & 0 & 0 & -1 & 0 & 0 & 0 & 0 & 1 & $w_{16}$ & 0 & 1 & 1 & 2 & 2 & 1 & 2 & 2 & 3 & 3 & 7 \\
$\boldsymbol{w}_{\mathbf{2}}$ & 0 & 0 & 0 & 0 & 1 & 0 & 0 & 0 & 0 & 1 & 1 & $w_{17}$ & 0 & 1 & 1 & 2 & 2 & 1 & 2 & 2 & 2 & 4 & 6 \\
$\boldsymbol{w}_{\mathbf{3}}$ & 0 & 0 & 1 & 1 & 1 & 1 & 1 & 1 & 1 & 1 & 1 & $w_{18}$ & 0 & 1 & 1 & 2 & 2 & 1 & 1 & 2 & 3 & 4 & 7 \\
$w_{4}$ & 0 & 1 & 1 & 1 & 1 & 1 & 1 & 1 & 2 & 2 & 1 & $\boldsymbol{w}_{19}$ & 0 & 1 & 1 & 2 & 3 & 2 & 2 & 3 & 3 & 3 & 6 \\
$w_{5}$ & 0 & 0 & 0 & 1 & 1 & 0 & 0 & 1 & 1 & 1 & 3 & $\boldsymbol{w}_{20}$ & 0 & 1 & 1 & 2 & 3 & 2 & 2 & 2 & 3 & 4 & 7 \\
$w_{6}$ & 0 & 0 & 0 & 1 & 1 & 0 & 0 & 0 & 1 & 2 & 3 & $w_{21}$ & 0 & 1 & 1 & 2 & 3 & 1 & 2 & 3 & 3 & 4 & 13 \\
$w_{7}$ & 0 & 0 & 1 & 1 & 1 & 0 & 0 & 1 & 2 & 2 & 3 & $w_{22}$ & 0 & 1 & 1 & 2 & 3 & 1 & 2 & 2 & 3 & 5 & 12 \\
$w_{8}$ & 0 & 0 & 1 & 1 & 1 & 0 & 1 & 1 & 1 & 2 & 3 & $w_{23}$ & 0 & 1 & 2 & 2 & 3 & 2 & 3 & 3 & 3 & 4 & 9 \\
$\boldsymbol{w}_{9}$ & 0 & 1 & 1 & 2 & 2 & 2 & 2 & 2 & 2 & 3 & 3 & $w_{24}$ & 0 & 1 & 2 & 2 & 3 & 2 & 2 & 3 & 4 & 4 & 9 \\
$\boldsymbol{w}_{10}$ & 0 & 0 & 1 & 1 & 2 & 1 & 1 & 1 & 2 & 2 & 4 & $w_{25}$ & 0 & 1 & 2 & 2 & 3 & 1 & 3 & 3 & 4 & 4 & 10 \\
$w_{11}$ & 0 & 0 & 1 & 1 & 2 & 0 & 0 & 2 & 2 & 3 & 5 & $w_{26}$ & 0 & 1 & 2 & 2 & 3 & 1 & 2 & 3 & 4 & 5 & 15 \\
$w_{12}$ & 0 & 0 & 1 & 1 & 2 & 0 & 1 & 2 & 2 & 2 & 8 & $\boldsymbol{w}_{27}$ & 0 & 1 & 2 & 3 & 4 & 3 & 3 & 4 & 4 & 5 & 12 \\
$w_{13}$ & 0 & 0 & 1 & 1 & 2 & 0 & 1 & 1 & 2 & 3 & 8 & $w_{28}$ & 0 & 1 & 2 & 3 & 4 & 2 & 3 & 4 & 5 & 5 & 20 \\
$w_{14}$ & 0 & 1 & 1 & 1 & 2 & 1 & 2 & 2 & 2 & 2 & 4 & $w_{29}$ & 0 & 1 & 2 & 3 & 4 & 1 & 3 & 4 & 5 & 6 & 22 \\
$w_{15}$ & 0 & 1 & 1 & 1 & 2 & 1 & 1 & 2 & 2 & 3 & 4 & & & & & & & & & &
\end{tabular}

Proof. We define a standard inequality to be an inequality of the form $r_{i}+r_{j} \leq s_{k}+m$, where $1 \leq i<j \leq 5,1 \leq k \leq 5$ and $m$ is a nonnegative integer. The condition that $(r, s) \in \mathscr{W}$ does not dominate $w_{v}$ is equivalent to a list of $\lambda_{v}$ standard inequalities, at least one of which must hold, where $\lambda_{v}$ is as given in the table. For example, $(r, s) \nsupseteq w_{1}$ if and only if $r_{1}+r_{2} \leq s_{1}$, whereas $(r, s) \nsupseteq w_{5}$ if and only if $r_{1}+r_{4} \leq s_{2}$ or $r_{4}+r_{5} \leq s_{2}+1$ or $r_{4}+r_{5} \leq s_{5}$. (We have used the conditions $r_{1} \leq \cdots \leq r_{5}$ and $s_{1} \leq \cdots \leq s_{5}$ to remove redundant inequalities.)

We wrote a program using the simplex algorithm to maximise $\sum\left(2 r_{i}-s_{i}\right)$ for $(r, s) \in \mathbb{R}^{10}$ subject to $0 \leq r_{1} \leq \cdots \leq r_{5}, 0 \leq s_{1} \leq \cdots \leq s_{5}$ and a list of standard 
inequalities. Our program starts with the basic feasible solution $(r, s)=(0,0)$. If there is a finite maximum and it is less than 1 , then (by definition of $\mathscr{W}$ ) there are no weights satisfying these inequalities. If the maximum is 1 , then we add the constraint $\sum\left(2 r_{i}-s_{i}\right)=1$. We then use the simplex algorithm to maximise each of the functions $r_{i}+r_{j}-s_{k}$ in turn. In the case of a finite maximum $\alpha$, we obtain an additional standard inequality $r_{i}+r_{j} \leq s_{k}+\max (\lfloor\alpha\rfloor, 0)$. Then running our original program on the enlarged set of standard inequalities, we may still be able to show that $\sum\left(2 r_{i}-s_{i}\right)<1$.

After processing the inequalities coming from $w_{1}, \ldots, w_{v}$ for $v=1, \ldots, 29$, the number of cases remaining were

$1,1,1,1,3,5,8,13,16,30,31,49,58,47,60$,

$$
64,58,53,45,36,39,34,25,15,14,10,3,1,0 .
$$

The final 0 indicates that no cases remain, and this proves the lemma. The proof of Lemma 5.4 is similar but easier.

If $\Phi \in X_{5}\left(\mathscr{O}_{K}\right)$ is nonminimal, then by Lemmas 5.2 and 6.1 it has slope at most 14 . This already shows that the algorithm in Theorem 3.1(iii) takes at most fourteen iterations. The next lemma improves this bound to seven iterations.

Lemma 6.2. If the procedure in Theorem 3.1(ii) returns a saturated model with the same level, then the slope goes down by at least 2.

Proof. We revisit the proof of Theorem 3.1(iii) at the end of Section 5. If the slope goes down by only 1 , then $\operatorname{Sing} \mathscr{C}_{\phi}$ spans a hyperplane. If $\operatorname{Sing} \mathscr{C}_{\phi}$ is a hyperplane, then the proof of Theorem 3.1(ii) at the end of Section 4 shows that the level is decreased. Otherwise, by Lemma 4.2 we may assume $\phi$ takes the form (5). We then follow the proof of Lemma 4.3(i) with $m=4$. After applying the transformation suggested there, the second row of $\phi$ has at most one nonzero entry. This implies that $\Phi$ is nonsaturated.

The next lemma will be used to show that only five iterations are required.

Lemma 6.3. Let $\Phi \in X_{5}\left(\mathscr{O}_{K}\right)$ be nonminimal and of slope greater than 10. Then replacing $\Phi$ by an $0_{K}$-equivalent model, we may assume it has weight $w_{29}$ and the coefficient of $x_{k}$ in $\Phi_{i j}$ is a unit for

$(i, j, k) \in$

$$
\{(1,2,1),(1,4,2),(1,5,3),(2,3,2),(2,4,3),(2,5,4),(3,4,4),(3,5,5)\} .
$$

Proof. By Lemma 5.2, we know that $\Phi$ is $\mathscr{O}_{K}$-equivalent to a model with one of the twenty-nine weights listed in Lemma 6.1. For all but one of these weights $(r, s)$, we have $\sum_{i=1}^{5}\left(s_{i}-s_{1}\right) \leq 10$. The remaining case is $w_{29}$. If one of the coefficients listed is not a unit, then $\Phi$ has weight $w_{v}$ for some $v \in\{1,5,13,26,16,21,8,12\}$. 
We write $[j, \ldots, 5]$ for a linear combination of $x_{j}, \ldots, x_{5}$ and underline in cases where we know the coefficient is nonzero. If the slope is at most 10 , then at most five iterations are needed. Thus, Lemma 6.3 shows that we can reduce to the case where $\Phi \in X_{5}\left(0_{K}\right)$ has reduction $\phi \in X_{5}(k)$ of the form

$$
\left(\begin{array}{cccc}
0[\underline{1}, 2,3,4,5] & {[2,3,4,5]} & {[\underline{2}, 3,4,5]} & {[\underline{3}, 4,5]} \\
0 & {[\underline{2}, 3,4,5]} & {[\underline{3}, 4,5]} & {[\underline{4}, 5]} \\
& 0 & {[\underline{4}, 5]} & {[\underline{5}]} \\
& & 0 & 0 \\
& & & 0
\end{array}\right) .
$$

Let $\operatorname{Pf}(\phi)=\left(p_{1}, \ldots, p_{5}\right)$. By considering the partial derivatives of $p_{1}, p_{2}$ and $p_{4}$ with respect to $x_{1}, x_{2}$ and $x_{3}$, we see that if $P=\left(x_{1}: \cdots: x_{5}\right) \in \operatorname{Sing} \mathscr{C}_{\phi}$, then $x_{5}=0$. Then since $P \in \mathscr{C}_{\phi}$, we have $x_{4}=x_{3}=x_{2}=0$. So $(1: 0: \cdots: 0)$ is the unique singular point.

Our algorithm applies the transformation

$$
\left[\operatorname{Diag}\left(1,1,1, \pi^{-1}, \pi^{-1}\right), \operatorname{Diag}(1, \pi, \pi, \pi, \pi)\right] .
$$

The result is a model $\Phi$ with weight $w_{26}=(0,1,2,2,3 ; 1,2,3,4,5)$ whose reduction $\phi$ takes the form

$$
\left(\begin{array}{ccccc}
0 & {[\underline{1}]} & 0 & {[2,3,3,4,5]} & {[\underline{3}, 4,5]} \\
0 & 0 & {[\underline{3}, 4,5]} & {[\underline{4}, 5]} \\
& 0 & {[\underline{4}, 5]} & {[\underline{5}]} \\
& & 0 & {[5]} \\
& & & 0
\end{array}\right) .
$$

A calculation similar to that above shows that $\operatorname{Sing} \mathscr{C}_{\phi}=\left\{x_{3}=x_{4}=x_{5}=0\right\}$.

Our algorithm applies the transformation

$$
\left[\operatorname{Diag}(\pi, 1,1,1,1), \operatorname{Diag}\left(\pi^{-1}, \pi^{-1}, 1,1,1\right)\right] .
$$

The result is a model $\Phi$ with weight $w_{13}=(0,0,1,1,2 ; 0,1,1,2,3)$ whose reduction $\phi$ takes the form

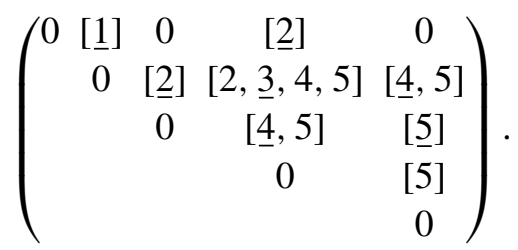

A calculation similar to that above shows that $\operatorname{Sing} \mathscr{C}_{\phi}=\left\{x_{2}=x_{4}=x_{5}=0\right\}$. 
The next transformation $\left[\operatorname{Diag}(1, \pi, 1,1,1), \operatorname{Diag}\left(\pi^{-1}, 1, \pi^{-1}, 1,1\right)\right]$ gives a model with weight $w_{15}=(0,1,1,1,2 ; 1,1,2,2,3)$. So after three iterations, the slope is at most 4 . It follows by Lemma 6.2 that at most five iterations are required.

Example 6.4. The simplest example of a genus-1 model satisfying the conditions of Lemma 6.3 is

$$
\Phi=\left(\begin{array}{ccccc}
0 & x_{1} & 0 & x_{2} & x_{3} \\
& 0 & x_{2} & x_{3} & x_{4} \\
& & 0 & x_{4} & x_{5} \\
& - & & 0 & 0 \\
& & & & 0
\end{array}\right)
$$

We find that $\mathscr{C}_{\Phi}$ is a rational curve with a cusp parametrised by

$$
(s: t) \mapsto\left(-s^{5}: s^{3} t^{2}: s^{2} t^{3}: s t^{4}: t^{5}\right) .
$$

In this case, our algorithm takes the maximum of exactly five iterations to give a nonsaturated model. (The first three iterations are already described above.) Although the model in this example is singular, there are $\pi$-adically close nonsingular models that are treated in the same way by our algorithm.

\section{Insoluble models}

In this section, we prove a result converse to the strong minimisation theorem. This is analogous to the results for models of degrees $n=2,3,4$ proved in [Cremona et al. 2010, Section 5]. As in Section 2, we work over a discrete valuation field $K$. We write $K^{\text {sh }}$ for the strict Henselisation of $K$. (If $K$ is a $p$-adic field, then this is the maximal unramified extension.)

Theorem 7.1. If $\Phi \in X_{5}(K)$ is nonsingular and $\mathscr{C}_{\Phi}\left(K^{\mathrm{sh}}\right)=\varnothing$, then the minimal level is at least 1 and is equal to 1 if $\operatorname{char}(k) \neq 5$.

As in Section 6, we write $[j, \ldots, 5]$ for a linear combination of $x_{j}, \ldots, x_{5}$ and underline in cases where we require the coefficient is nonzero.

Definition 7.2. A genus-1 model $\Phi \in X_{5}\left(\mathscr{O}_{K}\right)$ is critical if it has reduction $\bmod \pi$ of the form

$$
\left(\begin{array}{cccc}
0[\underline{1}, 2,3,4,5] & {[\underline{2}, 3,4,5]} & {[\underline{3}, 4,5]} & {[\underline{4}, 5]} \\
0 & {[\underline{3}, 4,5]} & {[\underline{4}, 5]} & {[\underline{5}]} \\
& 0 & {[\underline{5}]} & 0 \\
& & 0 & 0 \\
& & & 0
\end{array}\right)
$$

and $\pi^{-1} \Phi_{35}$ and $\pi^{-1} \Phi_{45}$ have reductions $\bmod \pi$ of the form $[\underline{1}, 2,3,4,5]$ and $[2,3,4,5]$. 
We show in the next three lemmas that critical models are insoluble, minimal and of positive level. We then take $K=K^{\text {sh }}$ and show that every insoluble model $\Phi \in X_{5}(K)$ is $K$-equivalent to a critical model.

Lemma 7.3. Critical models are insoluble over $K$.

Proof. Suppose $\left(x_{1}, \ldots, x_{5}\right) \in K^{5}$ is a nonzero solution with $\min \left\{v\left(x_{i}\right)\right\}=0$. By considering the $4 \times 4$ Pfaffians, we successively deduce $\pi\left|x_{5}, \pi\right| x_{4}, \ldots, \pi \mid x_{1}$. In particular, $\min \left\{v\left(x_{i}\right)\right\}>0$. This is the required contradiction.

Since the definition of a critical model is unchanged by an unramified field extension, it follows immediately that critical models are insoluble over $K^{\text {sh }}$.

\section{Lemma 7.4. Critical models are minimal.}

Proof. It is easy to see that critical models are saturated. Moreover, every point on $\mathscr{C}_{\phi}=\left\{x_{3}=x_{4}=x_{5}=0\right\}$ is singular. Our algorithm (see Theorem 3.1) makes the transformation $\left[\operatorname{Diag}(\pi, 1,1,1,1), \pi^{-1} \operatorname{Diag}(1,1, \pi, \pi, \pi)\right]$. This gives an integral model of the same level that is $\hat{O}_{K}$-equivalent (by a pair of cyclic permutation matrices) to a critical model.

If $\Phi$ were nonminimal, then our algorithm would succeed in reducing the level. But on the contrary, when given a critical model our algorithm endlessly cycles between five $\mathrm{O}_{K}$-equivalence classes.

The next lemma describes the possible levels of a critical model. To treat the cases $\operatorname{char}(k)=2,3$, we need to work with the $a$-invariants defined in Section 1 . Although these are not $\mathrm{SL}_{5} \times \mathrm{SL}_{5}$-invariant, if we make our choices of $a_{1}, b_{2}$ and $a_{3}$ so as not to introduce any new monomials when we lift to characteristic 0 , then they will be invariant under all pairs of diagonal matrices. It follows by the proof of Lemma 1.2 that $a_{1}, \ldots, a_{6}$ are isobaric, i.e.,

$$
a_{i} \circ\left[\operatorname{Diag}\left(\lambda_{1}, \ldots, \lambda_{5}\right), \operatorname{Diag}\left(\mu_{1}, \ldots, \mu_{5}\right)\right]=\left(\prod \lambda_{v}\right)^{2 i}\left(\prod \mu_{v}\right)^{i} a_{i} .
$$

Lemma 7.5. The level of a critical model is at least 1 and equal to 1 if $\operatorname{char}(k) \neq 5$.

Proof. Applying

$$
\left[\operatorname{Diag}\left(1, \pi^{-1 / 5}, \pi^{-2 / 5}, \pi^{-3 / 5}, \pi^{-4 / 5}\right), \operatorname{Diag}\left(\pi^{1 / 5}, \pi^{2 / 5}, \pi^{3 / 5}, \pi^{4 / 5}, \pi\right)\right]
$$

to a critical model $\Phi$ gives a model with coefficients in $\mathcal{O}_{K}\left[\pi^{1 / 5}\right]$. It follows by the isobaric property that $\pi^{i} \mid a_{i}(\Phi)$ for all $i$. Hence, $\Phi$ has positive level. 
The model with coefficients in $\mathscr{O}_{K}\left[\pi^{1 / 5}\right]$ has reduction

$$
\left(\begin{array}{ccccc}
0 & \lambda_{1} x_{1} & \mu_{2} x_{2} & -\mu_{3} x_{3} & -\lambda_{4} x_{4} \\
& 0 & \lambda_{3} x_{3} & \mu_{4} x_{4} & -\mu_{5} x_{5} \\
& & 0 & \lambda_{5} x_{5} & \mu_{1} x_{1} \\
& & & 0 & \lambda_{2} x_{2} \\
& & & & 0
\end{array}\right)
$$

for some $\lambda_{1}, \ldots, \lambda_{5}, \mu_{1} \ldots, \mu_{5} \in k^{\times}$. The invariants of this model are

$$
\begin{aligned}
& c_{4}(\lambda, \mu)=\lambda^{4}+228 \lambda^{3} \mu+494 \lambda^{2} \mu^{2}-228 \lambda \mu^{3}+\mu^{4}, \\
& c_{6}(\lambda, \mu)=-\lambda^{6}+522 \lambda^{5} \mu+10005 \lambda^{4} \mu^{2}+10005 \lambda^{2} \mu^{4}-522 \lambda \mu^{5}-\mu^{6}
\end{aligned}
$$

and $\Delta(\lambda, \mu)=\lambda \mu\left(\lambda^{2}-11 \lambda \mu-\mu^{2}\right)^{5}$, where $\lambda=\prod \lambda_{i}$ and $\mu=\prod \mu_{i}$. Computing a resultant shows that if $\operatorname{char}(k) \neq 5$, then $c_{4}(\lambda, \mu)$ and $\Delta(\lambda, \mu)$ have no common roots. Therefore, the critical model $\Phi$ with which we started satisfies either $v\left(c_{4}(\Phi)\right)=4$ or $v(\Delta(\Phi))=12$. It follows that $\Phi$ has level at most 1 .

Remark 7.6. The following example of a critical model of level 2 over $K=\mathbb{Q}_{5}$ shows that the hypothesis $\operatorname{char}(k) \neq 5$ cannot be removed from Lemma 7.5:

$$
\left(\begin{array}{ccccc}
0 & x_{1} & x_{2} & -x_{3} & -x_{4} \\
& 0 & x_{3} & x_{4} & -x_{5} \\
& & 0 & x_{5} & 35 x_{1} \\
& - & & 0 & 5 x_{2} \\
& & & & 0
\end{array}\right) .
$$

We recall that the minimal level is unchanged by an unramified field extension. Replacing $K$ by $K^{\text {sh }}$, we may assume for the rest of this section that $K$ is Henselian and its residue field $k$ is algebraically closed. To complete the proof of Theorem 7.1, we show the following:

Theorem 7.7. If $\Phi \in X_{5}\left(\mathscr{O}_{K}\right)$ is minimal and $\mathscr{C}_{\Phi}(K)=\varnothing$, then $\Phi$ is $\mathscr{O}_{K}$-equivalent to a critical model.

We start the proof of Theorem 7.7 with the following lemma:

Lemma 7.8. If $\Phi \in X_{5}\left(\mathscr{O}_{K}\right)$ is minimal, then its reduction $\phi \in X_{5}(k)$ has the following properties:

(i) the $4 \times 4$ Pfaffians of $\phi$ are linearly independent,

(ii) the subscheme $\mathscr{C}_{\phi} \subset \mathbb{P}^{4}$ does not contain a plane and

(iii) the entries of $\phi$ span the space of linear forms on $\mathbb{P}^{4}$.

Proof. (i) This follows by Theorem 3.2 and Lemma 3.3(i). 
(ii) Suppose $\mathscr{C}_{\phi}$ contains the plane $\left\{x_{4}=x_{5}=0\right\}$. By Lemma 3.4, we may assume the reduction $\bmod \pi$ of $\left[I_{5}, \operatorname{Diag}(1,1,1, \pi, \pi)\right] \Phi$ takes one of the two forms given in the lemma. We decrease the level by applying either [Diag $\left.(\pi, 1,1,1,1), \pi^{-1} I_{5}\right]$ or $\left[\operatorname{Diag}\left(1,1,1, \pi^{-1}, \pi^{-1}\right), B\right]$, where $B$ is chosen to preserve integrality.

(iii) This is clear, as we could otherwise decrease the level by dividing one of the coordinates by $\pi$.

Lemma 7.9. Let $\phi \in X_{5}(k)$ be a genus-1 model satisfying the conclusions of Lemma 7.8. Suppose that every point on $\mathscr{C}_{\phi}$ is singular. Then $\phi$ is $k$-equivalent to

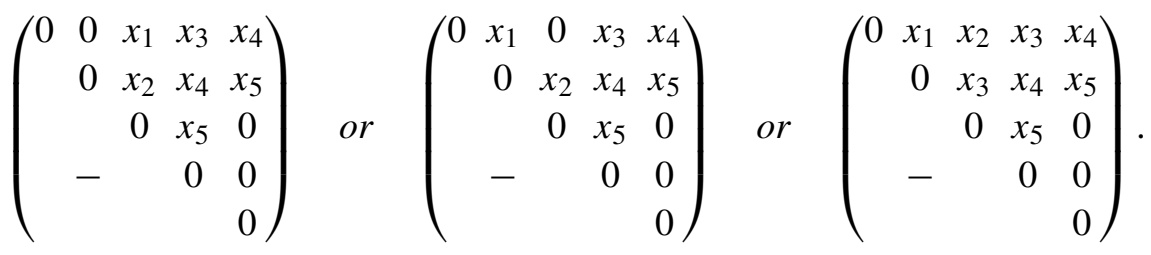

Our proof of Lemma 7.9 uses the following classification of degenerations of the twisted cubic. (Only the last sentence of the statement is needed.)

Lemma 7.10. Let $\psi$ be a $3 \times 2$ matrix of linear forms in $R=k\left[x_{1}, \ldots, x_{4}\right]$. Suppose the $2 \times 2$ minors of $\psi$ are linearly independent and no linear combination of them has rank 1 . Then $\psi$ is $\mathrm{GL}_{2} \times \mathrm{GL}_{3} \times \mathrm{GL}_{4}$-equivalent to one of the following:

$$
\left(\begin{array}{ll}
x_{1} & x_{2} \\
x_{2} & x_{3} \\
x_{3} & x_{4}
\end{array}\right), \quad\left(\begin{array}{ll}
x_{1} & x_{2} \\
x_{2} & x_{3} \\
x_{4} & 0
\end{array}\right), \quad\left(\begin{array}{cc}
x_{1} & x_{2} \\
0 & x_{3} \\
x_{4} & 0
\end{array}\right) \text { or }\left(\begin{array}{cc}
x_{1} & 0 \\
x_{2} & x_{2} \\
0 & x_{3}
\end{array}\right) \text {. }
$$

In particular, the locus of smooth points on $\Gamma=\{\operatorname{rank} \psi \leq 1\} \subset \mathbb{P}^{3}$ spans $\mathbb{P}^{3}$.

Proof. We may realise $\Gamma$ as the intersection of the image of the Segre embedding $\mathbb{P}^{1} \times \mathbb{P}^{2} \rightarrow \mathbb{P}^{5}$ with a linear subspace $\mathbb{P}^{3}$. So every component of $\Gamma$ has dimension at least 1 . If every component has dimension 1, then by the Buchsbaum-Eisenbud acyclicity criterion, there is a minimal free resolution

$$
0 \rightarrow R(-3)^{2} \stackrel{\psi}{\rightarrow} R(-2)^{3} \stackrel{M}{\rightarrow} R
$$

where $M$ is the vector of $2 \times 2$ minors of $\psi$. If in addition $\operatorname{dim} T_{P} \Gamma=1$ for every $P \in \Gamma$, then by an argument using Serre's criterion [Eisenbud 1995, Section 18.3], the ideal in $R$ generated by the $2 \times 2$ minors of $\psi$ is a prime ideal. By (10), the Hilbert polynomial is

$$
h(t)=\left(\begin{array}{c}
t+3 \\
3
\end{array}\right)-3\left(\begin{array}{c}
t+1 \\
3
\end{array}\right)+2\left(\begin{array}{l}
t \\
3
\end{array}\right)=3 t+1 .
$$

Therefore, $\Gamma$ is a twisted cubic and $\psi$ is equivalent to the first of the matrices in (9). 
In all other cases, $\operatorname{dim} T_{P} \Gamma>1$ for some $P \in \Gamma$. First suppose $\operatorname{rank} \psi(P)=1$. Moving $P$ to $(1: 0: 0: 0)$, we may suppose

$$
\psi=\left(\begin{array}{cc}
x_{1} & \alpha \\
\delta & \beta \\
\gamma & 0
\end{array}\right),
$$

where $\alpha, \beta, \gamma$ and $\delta$ are linear forms in $x_{2}, x_{3}, x_{4}$. Our hypotheses on the $2 \times 2$ minors ensure that $\alpha, \beta$ and $\gamma$ are linearly independent; say they are $x_{2}, x_{3}$ and $x_{4}$. By row and column operations (and a substitution for $x_{1}$ ), we may assume $\delta$ is a multiple of $x_{2}$. This gives the second and third cases in (9).

Now suppose rank $\psi(P)=0$. Let $Q \in \Gamma$ be any other point. If $\operatorname{rank} \psi(Q)=0$, then the $2 \times 2$ minors are binary quadratic forms, and so some linear combination has rank 1 . Therefore, $\operatorname{rank} \psi(Q)=1$. If $\operatorname{dim} T_{Q} \Gamma>1$, then our earlier analysis applies (and in fact gives a contradiction). Otherwise, we may assume

$$
\psi=\left(\begin{array}{cc}
x_{1} & 0 \\
\alpha & x_{2} \\
\beta & x_{3}
\end{array}\right),
$$

where $\alpha$ and $\beta$ are linear forms in $x_{2}, x_{3}$. (The 0 in the top right has been cleared by row operations.) Since $\alpha x_{3}-\beta x_{2}$ is a rank-2 quadratic form in $x_{2}, x_{3}$, we can make a change of coordinates so that $\Gamma=\left\{x_{1} x_{2}=x_{1} x_{3}=x_{2} x_{3}=0\right\}$. Then $\psi$ is equivalent to the last of the matrices in (9).

For the final statement, we note that the four cases correspond geometrically to (i) a twisted cubic, (ii) a conic and a line, (iii) three nonconcurrent lines and (iv) three concurrent lines. In each case, $\Gamma$ spans $\mathbb{P}^{3}$, and the only singular points are the points where the components meet.

Proof of Lemma 7.9. Let $P \in \mathscr{C}_{\phi}$ be a singular point. Moving $P$ to $(1: 0: 0: 0: 0)$, we may assume $\phi$ takes the form

$$
\left(\begin{array}{ccccc}
0 & x_{1} & \ell_{2} & \alpha_{1} & \beta_{1} \\
& 0 & \ell_{3} & \alpha_{2} & \beta_{2} \\
& & 0 & \alpha_{3} & \beta_{3} \\
& - & & 0 & 0 \\
& & & & 0
\end{array}\right)
$$

where $\ell_{i}, \alpha_{i}$ and $\beta_{i}$ are linear forms in $x_{2}, \ldots, x_{5}$. Let $\psi$ be the top right $3 \times 2$ submatrix, and let $\Gamma \subset \mathbb{P}^{3}$ be the curve defined by its $2 \times 2$ minors. Since the $2 \times 2$ minors of $\psi$ are a subset of the $4 \times 4$ Pfaffians of $\phi$, they are linearly independent. In particular, $\alpha_{3}$ and $\beta_{3}$ cannot both vanish identically. Without loss of generality, $\alpha_{3}$ is nonzero. 
Suppose no linear combination of the $2 \times 2$ minors of $\psi$ has rank 1 . Then by Lemma 7.10, there is a smooth point $Q=\left(x_{2}: x_{3}: x_{4}: x_{5}\right)$ on $\Gamma$ with $\alpha_{3}(Q) \neq 0$. Solving for $x_{1}$ gives a smooth point $\left(x_{1}: x_{2}: \cdots: x_{5}\right)$ on $\mathscr{C}_{\phi}$. This is a contradiction. Therefore, some linear combination of the $2 \times 2$ minors of $\psi$ has rank 1 . It is then easy to see that $\phi$ is $k$-equivalent to a model of the form (5).

By properties (i) and (ii), $\eta$ and $x_{5}$ are linearly independent, and $\gamma, \delta$ and $x_{5}$ are linearly independent. However, if $\eta, \gamma, \delta$ and $x_{5}$ were linearly independent, then taking them to be $x_{2}, \ldots, x_{5}$ would give that $(0: 1: 0: 0: 0)$ is a smooth point on $\mathscr{b}_{\phi}$. By row and column operations, we may therefore suppose $\eta=\delta\left(=x_{4}\right.$, say).

By property (ii), $\beta, x_{4}$ and $x_{5}$ are linearly independent, and $\gamma, x_{4}$ and $x_{5}$ are linearly independent. By row and column operations (and substitutions for the $x_{i}$ ), we may suppose $\beta=x_{3}$ and $\gamma=x_{2}$ or $x_{3}$. If $\gamma=x_{2}$, then by further row and column operations (and substitutions for the $x_{i}$ ), we may suppose $\alpha$ is a multiple of $x_{1}$. The lemma now follows using property (iii).

Proof of Theorem 7.7. Since $K$ is Henselian, any smooth point on $\mathscr{C}_{\phi}$ lifts to a $K$-point on $\mathscr{C}_{\Phi}$. So we may assume $\phi$ takes one of the three forms in Lemma 7.9. In the first two cases, $\phi$ defines a pair of concurrent lines with multiplicities 2 and 3 . (These cases may be distinguished by the dimension of the tangent space at the point of intersection.) In the third case, it defines a line with multiplicity 5.

We apply the transformation $\left[\operatorname{Diag}\left(1,1,1,1, \pi^{-1}\right), \operatorname{Diag}(1,1,1, \pi, \pi)\right]$. This gives an integral model of the same level. So the reduction must again be $k$ equivalent to one of the three models in Lemma 7.9. We tidy up by an $\mathrm{O}_{K^{-}}$ equivalence that cyclically permutes the rows and columns and makes substitutions for $x_{4}$ and $x_{5}$. The reduction $\phi \in X_{5}(k)$ now takes the form

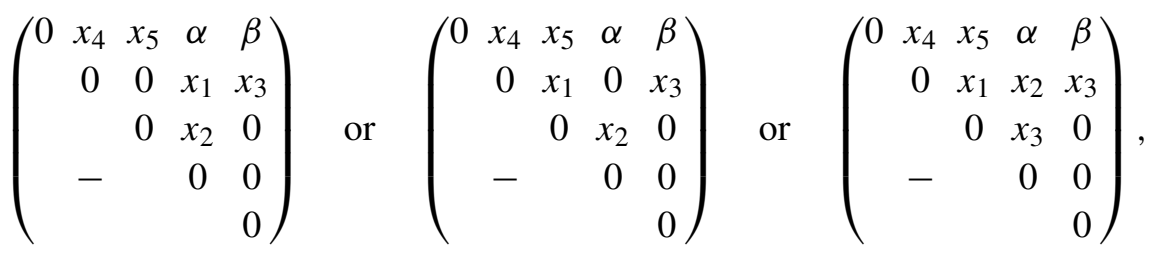

where $\alpha$ and $\beta$ are linear forms in $x_{1}, x_{2}, x_{3}$.

In the first case, $(0: 0: 0: 1: 0)$ is a point with tangent space of dimension 3 , and $\mathscr{b}_{\phi}$ contains points not on the line $\left\{x_{1}=x_{2}=x_{3}=0\right\}$. So the transformation has moved us to the second case.

In the second case, we obtain a contradiction as follows. If $\alpha=x_{1}+\lambda x_{2}+\mu x_{3}$, then adding $\mu$ times the fifth row/column to the third row/column and making substitutions for $x_{1}$ and $x_{5}$, we may assume $\mu=0$. Then $(0: 0: 1: 0: 0)$ is a smooth point on $\mathscr{C}_{\phi}$. Likewise, if $\beta=x_{1}+\lambda x_{2}+\mu x_{3}$, then subtracting $\lambda$ times the fourth row/column from the second row/column and making substitutions for $x_{1}$ and $x_{4}$, we may assume $\lambda=0$. Then $(0: 1: 0: 0: 0)$ is a smooth point on $\mathscr{C}_{\phi}$. We are forced 
to the conclusion that neither $\alpha$ nor $\beta$ involves $x_{1}$. But then $\mathscr{C}_{\phi}$ contains the plane $\left\{x_{2}=x_{3}=0\right\}$, and by Lemma 7.8, this contradicts that $\Phi$ is minimal.

In the third case, we show that if the transformation above brings us back to the third case, then the original model is critical. If $\beta=x_{1}+\lambda x_{2}+\mu x_{3}$, then adding $\lambda$ times the fourth row/column to the third row/column and making substitutions for $x_{1}$ and $x_{5}$, we may assume $\lambda=0$. Then $\mathscr{b}_{\phi}$ contains the lines $\left\{x_{1}=x_{2}=x_{3}=0\right\}$ and $\left\{x_{1}=x_{3}=x_{5}=0\right\}$. So if the transformation returns us to third case, then $\beta$ cannot involve $x_{1}$. Since $\mathscr{C}_{\phi}$ does not contain a plane and the $4 \times 4$ Pfaffians of $\phi$ are linearly independent, $\alpha$ must involve $x_{1}$ and $\beta$ must involve $x_{2}$. It follows by Definition 7.2 that the original model is $\mathbb{O}_{K}$-equivalent to a critical model.

\section{Reduction}

Let $C \subset \mathbb{P}^{4}$ be a genus-1 normal curve of degree 5 defined over $\mathbb{Q}$. We may represent it by a nonsingular genus- 1 model $\Phi \in X_{5}(\mathbb{Z})$. Running the algorithm in Section 3 locally at $p$ for all primes $p$ dividing the discriminant $\Delta(\Phi)$, we obtain a $\mathbb{Q}$-equivalent model (still with coefficients in $\mathbb{Z}$ ) whose discriminant is minimal in absolute value. If $C$ is everywhere locally soluble, then this discriminant is the minimal discriminant of $E=\operatorname{Jac}(C)$. It remains to make a $\mathrm{GL}_{5}(\mathbb{Z})$ change of coordinates on $\mathbb{P}^{4}$ so that (after running the LLL algorithm on the space of five quadrics defining the curve) the coefficients (and not just the invariants) are small. The general method, described in [Cremona et al. 2010, Section 6], is to run the LLL algorithm on the Gram matrix for the (unique) Heisenberg invariant inner product. In this section, we outline how to compute this inner product in the case $n=5$.

We recall that the Heisenberg group is the subgroup of $\mathrm{SL}_{5}(\mathbb{C})$ consisting of matrices $M_{T}$ that describe the action of $T \in E[5]$ on $C \subset \mathbb{P}^{4}$ by translation. For $T \neq 0_{E}$, we call the five points in $\mathbb{P}^{4}$ fixed by $M_{T}$ a syzygetic 5 -tuple. It may be shown (for example, by adapting the proof of [Fisher 2012, Proposition 4.1] or using that $H^{1}(\mathbb{R}, E[5])$ is trivial) that $\Phi$ is $\mathrm{SL}_{5}(\mathbb{R}) \times \mathrm{SL}_{5}(\mathbb{R})$-equivalent to a model in Hesse form:

$$
\left(\begin{array}{ccccc}
0 & a x_{0} & b x_{1} & -b x_{2} & -a x_{3} \\
& 0 & a x_{2} & b x_{3} & -b x_{4} \\
& & 0 & a x_{4} & b x_{0} \\
& - & & 0 & a x_{1} \\
& & & & 0
\end{array}\right)
$$

The invariants of this model are

$$
\begin{aligned}
& c_{4}=a^{20}+228 a^{15} b^{5}+494 a^{10} b^{10}-228 a^{5} b^{15}+b^{20}, \\
& c_{6}=-a^{30}+522 a^{25} b^{5}+10005 a^{20} b^{10}+10005 a^{10} b^{20}-522 a^{5} b^{25}-b^{30}
\end{aligned}
$$


and $\Delta=D^{5}$, where $D=a b\left(a^{10}-11 a^{5} b^{5}-b^{10}\right)$. For a model in Hesse form, the Heisenberg group is generated by $\operatorname{Diag}\left(1, \zeta, \ldots, \zeta^{4}\right)$, where $\zeta$ is a primitive fifth root of unity, and a cyclic permutation matrix. Since these matrices are unitary, the Heisenberg invariant inner product is the standard inner product on $\mathbb{R}^{5}$.

The Hessian, introduced in [Fisher 2012], is an $\mathrm{SL}_{5} \times \mathrm{SL}_{5}$-equivariant polynomial map $H: X_{5} \rightarrow X_{5}$ with the property that the Hessian of (11) is of the same form with $a$ and $b$ replaced by $-\partial D / \partial b$ and $\partial D / \partial a$.

Theorem 8.1. Let $\Phi \in X_{5}(\mathbb{C})$ be a nonsingular genus-1 model with invariants $c_{4}$ and $c_{6}$. Let $A$ be the $3 \times 5$ matrix of quadrics such that $\lambda \Phi+\mu H(\Phi)$ has $4 \times 4$ Pfaffians

$$
\left\{\lambda^{2} A_{1 i}+\lambda \mu A_{2 i}+\mu^{2} A_{3 i} \mid i=1, \ldots, 5\right\} .
$$

Then $\mathscr{X}=\{\operatorname{rank} A \leq 1\} \subset \mathbb{P}^{4}$ consists of thirty points, and the syzygetic 5-tuples for $\mathscr{C}_{\Phi}$ are the fibres of the map $\alpha: \mathscr{X} \rightarrow \mathbb{P}^{2}$ given by the first (or indeed any) column of $A$. The image of $\alpha$ is the set of six points $(x: y: z) \in \mathbb{P}^{2}$ satisfying

$$
\operatorname{rank}\left(\begin{array}{cccc}
0 & 5 x & y & 6 c_{4} x+z \\
x & y & 6 c_{4} x-z & 8 c_{6} x \\
y & -z & 8 c_{6} x & 9 c_{4}^{2} x
\end{array}\right) \leq 2 .
$$

Proof. It suffices to prove this for $\Phi$ in Hesse form. Then $\mathscr{X}$ is defined by

$$
\operatorname{rank}\left(\begin{array}{ccccc}
x_{0}^{2} & x_{1}^{2} & x_{2}^{2} & x_{3}^{2} & x_{4}^{2} \\
x_{1} x_{4} & x_{0} x_{2} & x_{1} x_{3} & x_{2} x_{4} & x_{0} x_{3} \\
x_{2} x_{3} & x_{3} x_{4} & x_{0} x_{4} & x_{0} x_{1} & x_{1} x_{2}
\end{array}\right) \leq 1
$$

and by [Barth et al. 1987, Proposition 1] is a set of thirty points. Evaluating the columns of (13) at these points, we obtain $(1: 0: 0)$ and $\left(1: \zeta^{i}: \zeta^{-i}\right)$ for $i=0, \ldots, 4$. These are the points $(\xi: \eta: v) \in \mathbb{P}^{2}$ satisfying

$$
\operatorname{rank}\left(\begin{array}{cccc}
\xi & \eta & v & 0 \\
v & \xi & 0 & -\eta \\
0 & 0 & \eta & v
\end{array}\right) \leq 2
$$

The remaining statements follow by direct calculation. In particular, our description (12) of the image of $\alpha$ is checked by making the substitution

$$
\left(\begin{array}{l}
x \\
y \\
z
\end{array}\right)=\left(\begin{array}{ccc}
a b & b^{2} & -a^{2} \\
-a(\partial D / \partial a)+b(\partial D / \partial b) & -2 b(\partial D / \partial a) & -2 a(\partial D / \partial b) \\
-(\partial D / \partial b)(\partial D / \partial a) & (\partial D / \partial a)^{2} & -(\partial D / \partial b)^{2}
\end{array}\right)\left(\begin{array}{l}
\xi \\
\eta \\
v
\end{array}\right) .
$$

We note that this change of coordinates and the matrix relating the $3 \times 3$ minors of (12) and (14) each have determinant a constant times a power of $D$. 
After computing the Hessian exactly (using the algorithm in [Fisher 2012, Section 11]), we use Theorem 8.1 to compute the syzygetic 5-tuples numerically. We then compute a Gram matrix for the Heisenberg invariant inner product as follows.

Proposition 8.2. Let $C \subset \mathbb{P}^{4}$ be a genus-1 normal curve defined over $\mathbb{R}$.

(i) Exactly two of the syzygetic 5-tuples for $C$ are defined over $\mathbb{R}$, say

$$
Y=\left\{y_{i} y_{j}=0 \mid i<j\right\} \subset \mathbb{P}^{4} \text { and } Z=\left\{z_{i} z_{j}=0 \mid i<j\right\} \subset \mathbb{P}^{4},
$$

where $y_{0}, \ldots, y_{4}$ and $z_{0}, \ldots, z_{4}$ are linear forms in $\mathbb{C}\left[x_{0}, \ldots, x_{4}\right]$.

(ii) One of the 5-tuples in (i) has 5 real points, and the other has 1 real point. We may therefore arrange that $y_{0}, \ldots, y_{4}$ and $z_{0}$ have real coefficients and that the pairs $z_{1}, z_{4}$ and $z_{2}, z_{3}$ are complex conjugates.

(iii) The Heisenberg invariant quadratic form spans the 1-dimensional real vector space

$$
\left\langle y_{0}^{2}, \ldots, y_{4}^{2}\right\rangle \cap\left\langle z_{0}^{2}, z_{1} z_{4}, z_{2} z_{3}\right\rangle .
$$

Proof. For $C$ in Hesse form, we may take $y_{i}=x_{i}$ and $z_{i}=\sum_{j=0}^{4} \zeta^{i j} x_{j}$. In this case, the Heisenberg invariant quadratic form is $x_{0}^{2}+\cdots+x_{4}^{2}$.

\section{Examples}

Wuthrich [2001] constructed an element of order 5 in the Tate-Shafarevich group of the elliptic curve $E / \mathbb{Q}$ with Weierstrass equation

$$
y^{2}+x y+y=x^{3}+x^{2}-3146 x+39049 .
$$

His example (see also [Fisher 2008, Section 9]) is defined by the $4 \times 4$ Pfaffians of

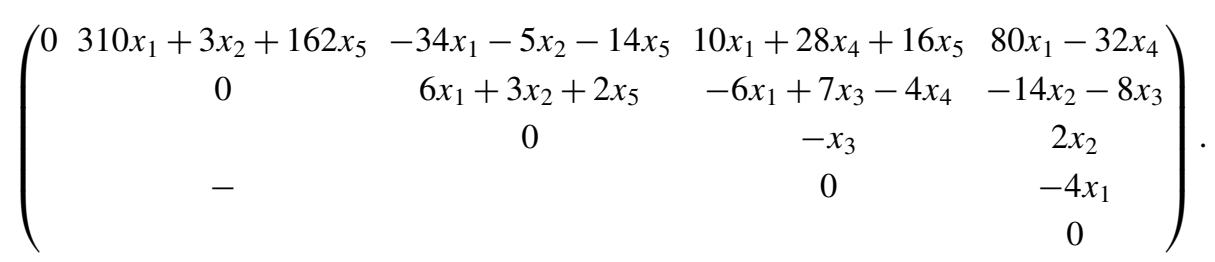

This model has discriminant $2^{132} \Delta_{E}$, where $\Delta_{E}$ is the minimal discriminant of $E$. In other words, the model is minimal at all primes except $p=2$, where the level is 11 . Minimisation and reduction suggest the change of coordinates

$$
\left(\begin{array}{l}
x_{1} \\
x_{2} \\
x_{3} \\
x_{4} \\
x_{5}
\end{array}\right) \leftarrow\left(\begin{array}{ccccc}
0 & 4 & -8 & 4 & 8 \\
0 & 0 & 0 & 0 & 16 \\
0 & -4 & 4 & 0 & 12 \\
4 & 5 & -15 & 2 & 7 \\
4 & -12 & 20 & -12 & -8
\end{array}\right)\left(\begin{array}{l}
x_{1} \\
x_{2} \\
x_{3} \\
x_{4} \\
x_{5}
\end{array}\right)
$$


so that Wuthrich's example simplifies to

$$
\Phi=\left(\begin{array}{ccccc}
0 & x_{2}+x_{5} & -x_{5} & -x_{1}+x_{2} & x_{4} \\
& 0 & x_{2}-x_{3}+x_{4} & x_{1}+x_{2}+x_{3}-x_{4}-x_{5} & x_{1}-x_{2}-x_{3}-x_{4}-x_{5} \\
& & 0 & x_{1}-x_{2}+2 x_{3}-x_{4}-x_{5} & -x_{2}-x_{4}+x_{5} \\
& & & 0 & -x_{3}-x_{4}-2 x_{5} \\
& & & & 0
\end{array}\right) .
$$

Our Magma function DoubleGenusOneModel, described in [Fisher 2013], computes a genus-1 model $\Phi^{\prime}$ that represents twice the class of $\Phi$ in the 5-Selmer group. This model has entries

$$
\begin{aligned}
& \Phi_{12}^{\prime}=3534132778 x_{1}+3583651940 x_{2}-881947110 x_{3}-323014538 x_{4}+3395115339 x_{5}, \\
& \Phi_{13}^{\prime}=5079379222 x_{1}-2965539950 x_{2}+11022202860 x_{3}+12821590868 x_{4}+640276471 x_{5}, \\
& \Phi_{14}^{\prime}=-10098238458 x_{1}-1274966110 x_{2}-7873816170 x_{3}-3456923272 x_{4}-62353929 x_{5}, \\
& \Phi_{15}^{\prime}=-12929747724 x_{1}-6790511810 x_{2}-11113305270 x_{3}-15161763156 x_{4}
\end{aligned}
$$$$
+3241937033 x_{5} \text {, }
$$$$
\Phi_{23}^{\prime}=-3381247332 x_{1}+3810679160 x_{2}+5919634530 x_{3}+75326852 x_{4}-1245085426 x_{5} \text {, }
$$$$
\Phi_{24}^{\prime}=-3572860258 x_{1}-5569480730 x_{2}-953739600 x_{3}-2138046812 x_{4}-858145244 x_{5} \text {, }
$$$$
\Phi_{25}^{\prime}=-4674149266 x_{1}-943631490 x_{2}-6754488160 x_{3}+751535046 x_{4}+117685567 x_{5},
$$$$
\Phi_{34}^{\prime}=-1851228934 x_{1}+5238146110 x_{2}-165588410 x_{3}-2070411506 x_{4}+678105748 x_{5} \text {, }
$$$$
\Phi_{35}^{\prime}=-6992835070 x_{1}-3744630360 x_{2}+3130208220 x_{3}-4523781310 x_{4}+433739425 x_{5} \text {, }
$$$$
\Phi_{45}^{\prime}=780078472 x_{1}+2039763820 x_{2}-450062790 x_{3}-7105731722 x_{4}+1625466111 x_{5} .
$$

The discriminant of $\Phi^{\prime}$ is $\Delta_{E}^{49}$. In particular, this model is nonminimal at all bad primes of $E$. Minimisation and reduction suggest the change of coordinates

$$
\left(\begin{array}{l}
x_{1} \\
x_{2} \\
x_{3} \\
x_{4} \\
x_{5}
\end{array}\right) \leftarrow\left(\begin{array}{ccccc}
92 & -36 & -153 & 129 & -131 \\
-54 & 84 & 5 & -206 & 139 \\
-63 & -174 & -60 & -79 & 53 \\
-111 & 106 & 206 & -115 & -162 \\
314 & -466 & 158 & -328 & -12
\end{array}\right)\left(\begin{array}{l}
x_{1} \\
x_{2} \\
x_{3} \\
x_{4} \\
x_{5}
\end{array}\right)
$$

so that $\Phi^{\prime}$ simplifies to

$$
\left(\begin{array}{cccc}
0-x_{4}+x_{5} & x_{3}-x_{4}+x_{5} & x_{2}-x_{5} & x_{1}-x_{2}+x_{3}-x_{4}-2 x_{5} \\
0 & x_{1}+x_{5} & -x_{2}-x_{3} & -x_{2}+x_{5} \\
& 0 & x_{4} & -x_{1} \\
- & & 0 & x_{1}+x_{4}-x_{5} \\
& & & 0
\end{array}\right) .
$$


See also [Creutz and Miller 2012, Section 7.4] for an example where our algorithms are used to help find a Mordell-Weil generator of large height.

\section{References}

[Artin et al. 2005] M. Artin, F. Rodriguez-Villegas, and J. Tate, "On the Jacobians of plane cubics", Adv. Math. 198:1 (2005), 366-382. MR 2006h:14043 Zbl 1092.14054

[Barth et al. 1987] W. Barth, K. Hulek, and R. Moore, "Shioda's modular surface $S(5)$ and the Horrocks-Mumford bundle”, pp. 35-106 in Vector bundles on algebraic varieties (Bombay, 1984), Tata Inst. Fund. Res. Stud. Math. 11, Oxford University Press, New York, 1987. MR 88j:14027 Zbl 0676.14010

[Bosma et al. 1997] W. Bosma, J. Cannon, and C. Playoust, “The Magma algebra system, I: The user language”, J. Symbolic Comput. 24:3-4 (1997), 235-265. MR 1484478 Zbl 0898.68039

[Cremona et al. 2010] J. E. Cremona, T. A. Fisher, and M. Stoll, "Minimisation and reduction of 2-, 3and 4-coverings of elliptic curves", Algebra Number Theory 4:6 (2010), 763-820. MR 2012c:11120 Zbl 1222.11073

[Creutz and Miller 2012] B. Creutz and R. L. Miller, "Second isogeny descents and the Birch and Swinnerton-Dyer conjectural formula", J. Algebra 372 (2012), 673-701. MR 2990032

[Eisenbud 1995] D. Eisenbud, Commutative algebra: with a view toward algebraic geometry, Graduate Texts in Mathematics 150, Springer, New York, 1995. MR 97a:13001 Zbl 0819.13001

[Fisher 2008] T. A. Fisher, "The invariants of a genus one curve", Proc. Lond. Math. Soc. (3) 97:3 (2008), 753-782. MR 2009j:11087 Zbl 1221.11135

[Fisher 2012] T. A. Fisher, "The Hessian of a genus one curve", Proc. Lond. Math. Soc. (3) 104:3 (2012), 613-648. MR 2900238 Zbl 06021282

[Fisher 2013] T. A. Fisher, "Invariant theory for the elliptic normal quintic, I: Twists of X(5)", Math. Ann. 356:2 (2013), 589-616. MR 3048608 Zbl 06181632

[Kraus 1989] A. Kraus, "Quelques remarques à propos des invariants $c_{4}, c_{6}$ et $\Delta$ d'une courbe elliptique”, Acta Arith. 54:1 (1989), 75-80. MR 90j:11045 Zbl 0628.14024

[Wuthrich 2001] C. Wuthrich, "Une quintique de genre 1 qui contredit le principe de Hasse", Enseign. Math. (2) 47:1-2 (2001), 161-172. MR 2002c:14037 Zbl 1064.14019

Communicated by Karl Rubin

Received 2012-02-02 Accepted 2012-08-20

T.A.Fisher@dpmms.cam.ac.uk Department of Pure Mathematics and Mathematical Statistics, University of Cambridge, Wilberforce Road, Cambridge, CB3 OWB, United Kingdom http://www.dpmms.cam.ac.uk/ taf1000/ 


\section{Algebra \& Number Theory}

msp.org/ant

\section{EDITORS}

MANAGING EDITOR

Bjorn Poonen

Massachusetts Institute of Technology

Cambridge, USA

\author{
EDITORIAL BOARD CHAIR \\ David Eisenbud \\ University of California \\ Berkeley, USA
}

\section{BOARD OF EDITORS}

Georgia Benkart

Dave Benson

Richard E. Borcherds

John H. Coates

J-L. Colliot-Thélène

Brian D. Conrad

Hélène Esnault

Hubert Flenner

Edward Frenkel

Andrew Granville

Joseph Gubeladze

Ehud Hrushovski

Craig Huneke

Mikhail Kapranov

Yujiro Kawamata

János Kollár

Yuri Manin

Barry Mazur

Philippe Michel
University of Wisconsin, Madison, USA

University of Aberdeen, Scotland

University of California, Berkeley, USA

University of Cambridge, UK

CNRS, Université Paris-Sud, France

University of Michigan, USA

Freie Universität Berlin, Germany

Ruhr-Universität, Germany

University of California, Berkeley, USA

Université de Montréal, Canada

San Francisco State University, USA

Hebrew University, Israel

University of Virginia, USA

Yale University, USA

University of Tokyo, Japan

Princeton University, USA

Northwestern University, USA

Harvard University, USA

École Polytechnique Fédérale de Lausanne
Susan Montgomery

Shigefumi Mori

Raman Parimala

Jonathan Pila

Victor Reiner

Karl Rubin

Peter Sarnak

Joseph H. Silverman

Michael Singer

Vasudevan Srinivas

J. Toby Stafford

Bernd Sturmfels

Richard Taylor

Ravi Vakil

Michel van den Bergh

Marie-France Vignéras

Kei-Ichi Watanabe

Efim Zelmanov
University of Southern California, USA

RIMS, Kyoto University, Japan

Emory University, USA

University of Oxford, UK

University of Minnesota, USA

University of California, Irvine, USA

Princeton University, USA

Brown University, USA

North Carolina State University, USA

Tata Inst. of Fund. Research, India

University of Michigan, USA

University of California, Berkeley, USA

Harvard University, USA

Stanford University, USA

Hasselt University, Belgium

Université Paris VII, France

Nihon University, Japan

University of California, San Diego, USA

\section{PRODUCTION}

production@msp.org

Silvio Levy, Scientific Editor

See inside back cover or msp.org/ant for submission instructions.

The subscription price for 2013 is US \$200/year for the electronic version, and \$350/year ( $\$ 40$, if shipping outside the US) for print and electronic. Subscriptions, requests for back issues and changes of subscribers address should be sent to MSP.

Algebra \& Number Theory (ISSN 1944-7833 electronic, 1937-0652 printed) at Mathematical Sciences Publishers, 798 Evans Hall \#3840, c/o University of California, Berkeley, CA 94720-3840 is published continuously online. Periodical rate postage paid at Berkeley, CA 94704, and additional mailing offices.

ANT peer review and production are managed by EditFLOW ${ }^{\circledR}$ from Mathematical Sciences Publishers.

\section{PUBLISHED BY}

- mathematical sciences publishers

nonprofit scientific publishing

http://msp.org/

(C) 2013 Mathematical Sciences Publishers 


\section{Algebra \& Number Theory}

Volume $7 \quad$ No. $5 \quad 2013$

Sharp upper bounds for the Betti numbers of a given Hilbert polynomial

GiUlio CAVIGLia and SATOShi Murai

Comparing numerical dimensions

1065

BRIAN LEHMANN

Some consequences of a formula of Mazur and Rubin for arithmetic local constants JAN NEKOVÁŘ

Quantized mixed tensor space and Schur-Weyl duality

RICHARD DIPPER, STEPHEN DOTY and FRIEDERIKE STOLL

Weakly commensurable $S$-arithmetic subgroups in almost simple algebraic groups of

types B and C

SKIP GARIBALDI and ANDREI RAPINCHUK

Minimisation and reduction of 5-coverings of elliptic curves

1179

TOM FISHER

On binary cyclotomic polynomials

1207

ÉTIENNE FOUVRY

Local and global canonical height functions for affine space regular automorphisms

SHU KAWAGUCHI

On the ranks of the 2-Selmer groups of twists of a given elliptic curve

1101

1121

147

DANIEL M. KANE 